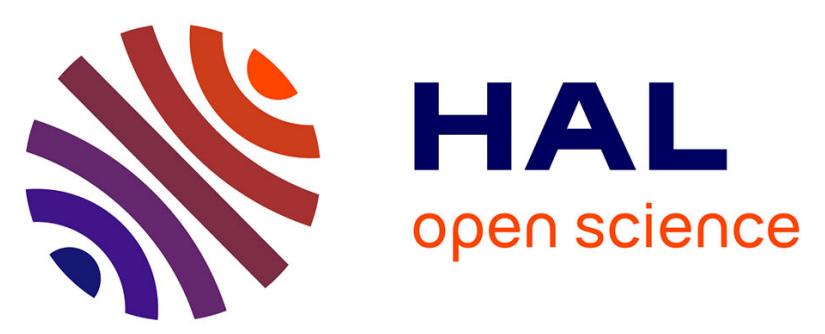

\title{
Simulation of Topography Effects on Rockfall-Generated Seismic Signals: Application to Piton de la Fournaise Volcano
}

\author{
Julian Kuehnert, Anne Mangeney, Y. Capdeville, Jean-Philippe Métaxian, \\ Luis Fabian Bonilla, Eleonore Stutzmann, E. Chaljub, Patrice Boissier, C. \\ Brunet, Philippe Kowalski, et al.
}

\section{To cite this version:}

Julian Kuehnert, Anne Mangeney, Y. Capdeville, Jean-Philippe Métaxian, Luis Fabian Bonilla, et al.. Simulation of Topography Effects on Rockfall-Generated Seismic Signals: Application to Piton de la Fournaise Volcano. Journal of Geophysical Research: Solid Earth, 2020, 125 (10), 10.1029/2020JB019874 . hal-03036404

\section{HAL Id: hal-03036404 https://hal.science/hal-03036404}

Submitted on 2 Dec 2020

HAL is a multi-disciplinary open access archive for the deposit and dissemination of scientific research documents, whether they are published or not. The documents may come from teaching and research institutions in France or abroad, or from public or private research centers.
L'archive ouverte pluridisciplinaire HAL, est destinée au dépôt et à la diffusion de documents scientifiques de niveau recherche, publiés ou non, émanant des établissements d'enseignement et de recherche français ou étrangers, des laboratoires publics ou privés. 


\title{
Simulation of topography effects on rockfall-generated seismic signals: application to Piton de la Fournaise volcano
}

\author{
J. Kuehnert ${ }^{1}$, A. Mangeney ${ }^{1}$, Y. Capdeville ${ }^{2}$, J. P. Métaxian ${ }^{1}$, L. F. Bonilla ${ }^{3}$, \\ E. Stutzmann ${ }^{1}$, E. Chaljub ${ }^{4}$, P. Boissier ${ }^{1,5}$, C. Brunet ${ }^{1,5}$, P. Kowalski ${ }^{1,5}$, F. \\ Lauret $^{1,5}$, Clément Hibert ${ }^{6}$ \\ ${ }^{1}$ Université de Paris, Institut de Physique du Globe de Paris, CNRS, F-75005 Paris, France \\ ${ }^{2}$ Laboratoire de Planétologie et Géodynamique, UMR CNRS 6112, Université de Nantes,44300 Nantes, \\ France \\ ${ }^{3}$ Université Gustave Eiffel, Marne-la-Vallée, France \\ ${ }^{4}$ Univ. Grenoble Alpes, Univ. Savoie Mont Blanc, CNRS, IRD, IFSTTAR, ISTerre, 38000 Grenoble, \\ France \\ ${ }^{5}$ Observatoire Volcanologique du Piton de la Fournaise/Institut de Physique du Globe de Paris, CNRS \\ UMS 3454 \& UMR 7154, Sorbonne Paris Cité, La Plaine des Cafres, Reunion Island, France \\ ${ }^{6}$ Université de Strasbourg, CNRS, EOST/IPGS UMR 7516, F-67000 Strasbourg, France
}

Key Points:

- First-ever simulation of high-frequency rockfall seismic waves using the 3D Spectral Element Method

- Ground-motion amplification induced by volcano topography found to be dependent on soil properties and rockfall position

- Simulations and observations successfully compared by means of inter-station spectral ratios and Hertz theory

Corresponding author: Julian Kuehnert, kuehnert@ipgp.fr 


\begin{abstract}
Seismic waves generated by rockfalls contain valuable information on the properties of these events. However, as rockfalls mainly occur in mountainous regions, the generated seismic waves can be affected by strong surface topography variations. We present a methodology for investigating the influence of topography using a Spectral-Element-based simulation of 3D wave propagation in various geological media. This methodology is applied here to Dolomieu crater on the Piton de la Fournaise volcano, Reunion Island, but it can be used for other sites, taking into account local topography and medium properties.

The complexity of wave fields generated by single-point forces is analyzed for different velocity models and topographies. Ground-motion amplification is studied relative to flat reference models, showing that Peak Ground Velocity (PGV) and total kinetic energy can be amplified by factors of up to 10 and 20, respectively. Simulations with Dolomieu-like crater shapes suggest that curvature variations are more influential than depth variations.

Topographic effects on seismic signals from rockfalls at Dolomieu crater are revealed by inter-station spectral ratios. Results suggest that propagation along the topography rather than source direction dominates the spectral ratios and that resulting radiation patterns can be neglected.

The seismic signature of single rockfall impacts is studied. Using Hertz contact theory, impact force and duration are estimated and then used to scale simulations, achieving order-of-magnitude agreement with observed signal amplitudes and frequency thresholds. Our study shows that combining Hertz theory with high-frequency seismic wave simulations on real topography improves the quantitative analysis of rockfall seismic signals.
\end{abstract}

\title{
1 Introduction
}

Interactions between seismic wave fields and complex surface geometries can locally modify seismic ground motion. Anomalously strong shaking on hilltops and mountain ridges or flanks, often causing severe structural damage to buildings (W. H. K. Lee et al., 1994; Hartzell et al., 1994; Hough et al., 2010) or triggering earthquake-induced landslides (Meunier et al., 2008; Harp et al., 2014), have been related to seismic amplification due to such topographic effects. Data from field experiments support the assumption of amplified ground motion at the top compared to the bottom of a mountain (Davis \& West, 1973; Pedersen et al., 1994; Spudich et al., 1996).

Numerous studies have tried to quantify numerically the topographic effect on seismic waves generated by deep sources. Geli et al. (1988) provided an extensive review of previous studies together with new results from more complex models (i.e. including subsurface layering and neighboring ridges). Using an earthquake simulation with three-dimensional topography, Bouchon and Barker (1996) found that a small hill of less than 20-m high can amplify ground acceleration by $30 \%$ to $40 \%$ for frequencies between $2 \mathrm{~Hz}$ and $15 \mathrm{~Hz}$. Using the 3D spectral element method, S. J. Lee, Chan, et al. (2009) studied the effects of high-resolution surface topography. They found that values of Peak Ground Acceleration (PGA) can be increased up to $100 \%$ relative to simulations on a flat surface and reported an increase in cumulative kinetic energy of up to $200 \%$ as a result of increased duration of shaking linked to complex reflection and scattering processes during the interaction of the seismic waves with the topography.

Yet, because of complex patterns of amplification and deamplification, it is difficult to quantify the effect of topography in a generic way. Maufroy et al. (2015) proposed to use the topography curvature, smoothed over a characteristic length depending on the studied wavelength, as a proxy for amplification factors. Based on the NGA-West2 earthquake catalog (Ancheta et al., 2014), Rai et al. (2017) showed statistical biases of site residuals in the ground-motion prediction equation (GMPE, Chiou \& Youngs, 2014) towards relative elevation and smoothed curvature and suggested topographic modification factors dependent on signal frequency and relative elevation. In addition to these 
successful findings, other authors have pointed out the complex coupling between topography and the underlying soil structure that must not be neglected when estimating topographic amplification (Assimaki \& Jeong, 2013; Hailemikael et al., 2016; B. Wang et al., 2018; Jeong et al., 2019).

All the studies mentioned above investigate topographic effects on a seismic wave field of vertical incidence. S. J. Lee, Komatitsch, et al. (2009) investigates the influence of the source depth on ground motion amplification and demonstrates that amplification in a basin can be reduced when a mountain range is located between the basin and a shallow source. This suggests that surface topography can have a pronounced influence on the propagation of surface waves subjected to an accumulated effect of scattering, diffraction, reflection, and conversion. It is crucial to enhance our understanding of these mechanisms for the study of shallow seismic sources that have gained increasing attention in the emerging field of environmental seismology (Larose et al., 2015). Several authors have investigated numerically the interaction of surface waves with 2D surface geometries such as corners, hills or canyons (Munasinghe \& Farnell, 1973; Weaver, 1982; Snieder, 1986; Sánchez-Sesma \& Campillo, 1993; Zhang et al., 2018; B. Wang et al., 2018). Ma et al. (2007) demonstrated that a topographic feature 10 times smaller than the wavelength can still considerably reduce the amplitude of by-passing surface waves. Similar to S. J. Lee, Komatitsch, et al. (2009), they simulated the shielding effects of large-scale topography on fault-generated surface waves using a 3D model of the San Gabriel Mountains, Los Angeles, California, finding amplification factors in peak ground velocity (PGV) of up to $+50 \%$ on the source-side of the mountain range and up to $-50 \%$ on the opposite site. L. Wang et al. (2015) modeled the influence of an uplifted and a depressed topography on the wave field. Comparing amplitudes and frequency content between source side and far-source side, they found that the depressed topography caused stronger contrasts than the uplifted topography, especially for steeper slopes and at higher frequencies.

The present study is focused on seismic waves generated by rockfalls. Different than the source mechanism of earthquakes, rockfall seismic sources can generally be described by impulse forces on the Earth's surface. Seismic signals from rockfalls, or more generally from landslides, have been demonstrated to be very useful to classify and locate events as well as constrain flow dynamics and rheology (e.g. Vilajosana et al., 2008; Deparis et al., 2008; Favreau et al., 2010; Hibert et al., 2011; Dammeier et al., 2011; Moretti et al., 2012; Bottelin et al., 2014). However, as landslides predominantly occur in areas of strong topographic relief, the measurements can be strongly influenced by topography variations leading to erroneous landslide estimates. For example, to calculate landslide volumes, the generated seismic energy is estimated from seismic recordings (Hibert et al., 2011). At the same time, energy estimations can vary from station to station. The present work shows that the topography studied here can partly explain amplitude variations between seismic stations.

In the following, after introducing the study site located at Dolomieu crater on Piton de la Fournaise volcano, Reunion Island, the numerical model for the SEM simulations is defined, entailing a discussion on the seismic velocity profile at Piton de la Fournaise. As the mesh size affects the computational cost, different topography resolutions are compared. Then, topography induced amplification is computed, depending on the underlying velocity model, by means of peak ground velocity (PGV) and total kinetic energy for both vertical and horizontal sources. In an attempt to quantify the dependencies on geometric parameters, the influence of variations in crater depth and curvature are investigated.

Finally, real seismic signals generated by rockfalls at Dolomieu crater are analyzed. Simulated and observed inter-station spectral ratios are compared, making it possible to examine the spectral content of the signals independently of the rockfall source. Additionally, the seismic signature of single rockfall impacts is investigated. To compare signal amplitude and frequency content between observations and simulations, impact force and duration are estimated based on Hertz contact theory (Hertz, 1878). 


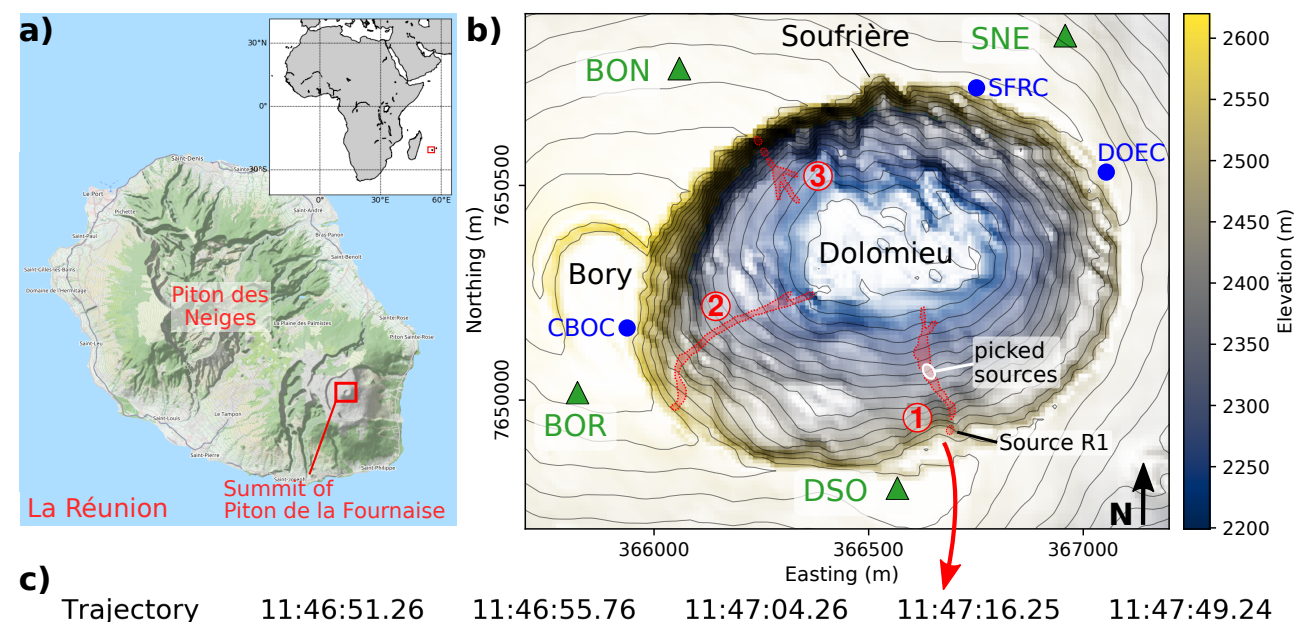

c)

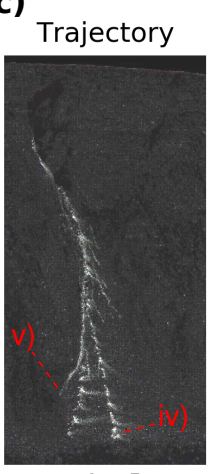

d)

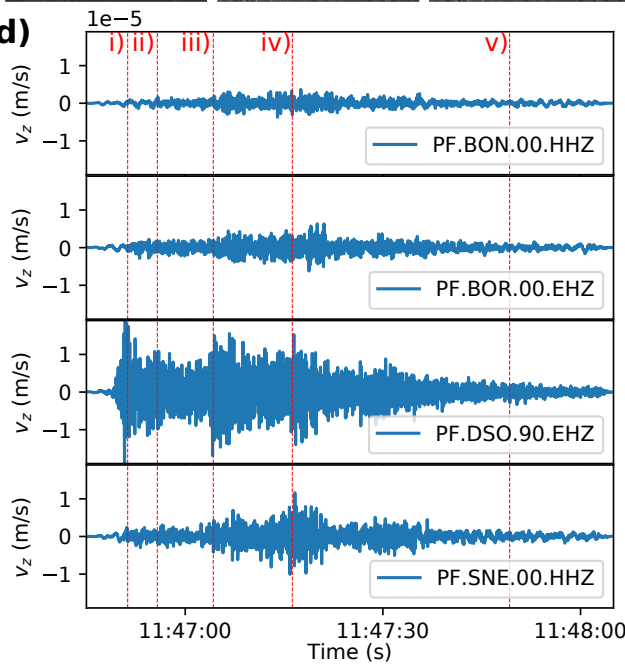

11:46:51.26

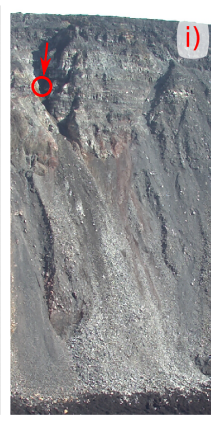

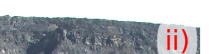

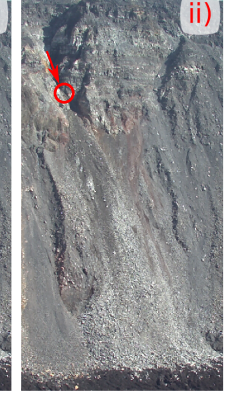

e)

e)
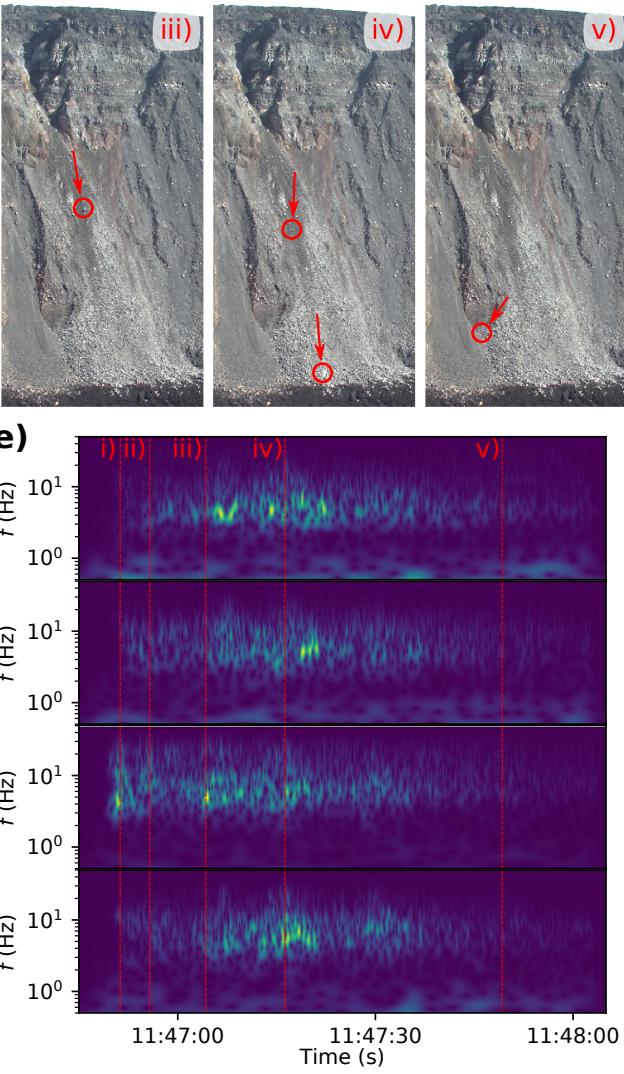

Figure 1. a) Map of Reunion Island with dormant volcano Piton des Neiges and active volcano Piton de la Fournaise. b) The summit of Piton de la Fournaise with $340 \mathrm{~m}$ deep Dolomieu crater and smaller craters Bory and Soufrière. Trajectories of three rockfalls are indicated by red zones. Seismic stations BON, BOR, DSO, and SNE (green triangles); cameras CBOC, DOEC, and SFRC (blue dots). Contour lines show elevation differences of $20 \mathrm{~m}$. c) Trajectory and snapshots from camera SFRC of rockfall 1 at the southern crater wall on February 28, 2016. Circles and arrows mark a selection of boulder positions and their direction of arrival. A video of the rockfall is provided in the Supporting Information. d) Vertical ground velocity recorded at all four stations. Vertical lines from i) to v) mark the times of camera snapshots in c). e) Corresponding spectrograms (Stockwell transform). 
$1800 \mathrm{~m}$ (northing), and $z=600 \mathrm{~m}$ (depth). To simulate an open domain, $160 \mathrm{~m}$ thick absorbing PML boundaries (Perfectly Matched Layers, e.g. Festa \& Vilotte, 2005) are added on the sides and bottom of the domain. The elements are successively deformed in the vertical direction to accommodate the topography provided by a Digital Elevation Model (DEM) with a $10 \mathrm{~m}$ resolution. To decrease computational costs, the element size is increased from $10 \mathrm{~m}$ to $30 \mathrm{~m}$ at $150 \mathrm{~m}$ below the surface (Zone of refinement), resulting in a total of 915,704 elements. To filter out short wavelength variations of the fine mesh that cannot be represented in the coarse mesh, a smooth Buffer layer, provided by a low-pass filtered topography, is used as an additional boundary $100 \mathrm{~m}$ below the surface. Simulations are implemented using a polynomial degree of 5, i.e. 6 GLL points (GaussLobatto-Legendre, e.g. Chaljub et al., 2007) per element in each direction.

Given the high computational costs (i.e. CPU times of up to 460 days, with 10 cores per CPU, for the heterogeneous velocity model), a mesh with a reduced topography resolution of $20 \mathrm{~m}$ is used in the first part of this study in which different velocity models are explored with a fixed point source located at the southern crater wall. This mesh is built using elements with a constant side length of $20 \mathrm{~m}$, which reduces the total number of elements to 550,000 , accordingly decreasing the CPU time to 145 days for the heterogeneous velocity model. The reduced number of elements also increases memory efficiency when displaying snapshots.

In the second part of the study, when comparing simulations to observations of rockfalls at Dolomieu crater, the model with high-resolution topography is used. Here we apply the reciprocity principle (Bettuzzi, 2009), i.e. the synthetic source is located at the position of the real seismometers (BON, BOR, DSO and SNE) and the wave field is recorded on a $10 \times 10 \mathrm{~m}$ grid of stations across Dolomieu crater. In this way, the impulse responses of all potential rockfall sources are modeled with just one simulation per seismometer and per channel.

The mesh of the flat-surface reference model is built with elements with $20 \mathrm{~m}$ side lengths. Cross-sections through all meshes (i.e. those with a flat surface; with $20 \mathrm{~m}$-resolution topography; with $10 \mathrm{~m}$-resolution topography) are provided in the Supporting Information.

\subsection{Velocity model}

Three different velocity models are used: (1) a homogeneous model, (2) a model with shallow low S-wave velocity layer, and (3) a model with smoothly increasing velocity as proposed by Lesage et al. (2018) for shallow volcano structures. The velocity-depth profiles are illustrated in Figure 2b and summarized in Table 1. The generic model by Lesage et al. (2018) is based upon measurements on multiple andesitic and basaltic volcanoes. P- and S-wave speeds $c_{i}$ are expressed as follows:

$$
c_{i}(z)=c_{i 0}\left[\left(z+a_{i}\right)^{\alpha_{i}}-a_{i}^{\alpha_{i}}+1\right], \quad i=P, S,
$$

where $z$ is the depth below the surface, $c_{i 0}$ are the velocities at zero depth, and $\alpha_{i}$ and $a_{i}$ are fitting parameters as defined in Table 1 .

The velocity profiles are compared to the S-wave velocity model inverted from ambient noise recordings at Piton de la Fournaise by Mordret et al. (2015). The orangeshaded zone shown in Figure $2 \mathrm{~b}$ corresponds to depth-profiles extracted from the inverted 3D model in the vicinity of Dolomieu crater. Good agreement is observed with the Lesage generic velocity profile. The discrepancy in the first $100 \mathrm{~m}$ can be caused by missing highfrequency content in the model of Mordret et al. (2015), who inverted frequencies below $2.5 \mathrm{~Hz}$.

In order to further validate the Lesage generic model for our study site, Rayleigh velocity dispersion curves from noise measurements at a mini-array located around station BON are compared in Figure 2c with theoretical dispersion curves of the Lesage generic model. Picks from the mini-array measurements are determined using the Modified Spatial Autocorrelation (MSPAC) Toolbox (Köhler et al., 2007; Wathelet et al., 2008) as im- 
a)

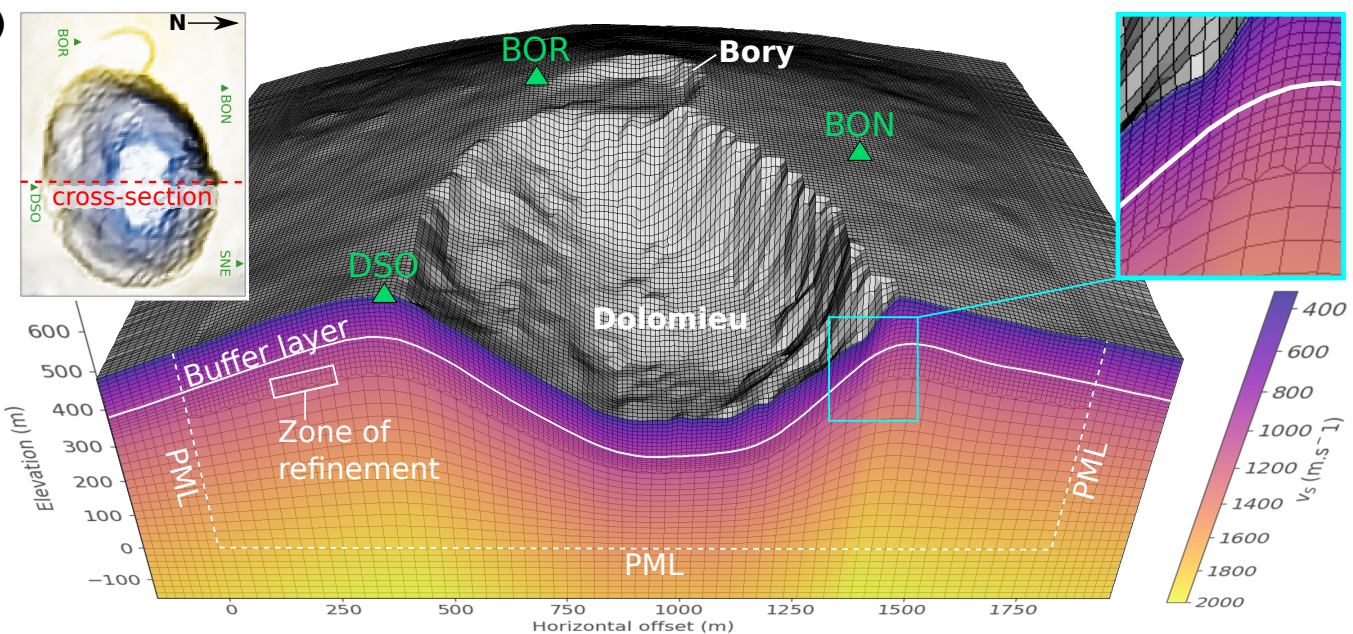

b)

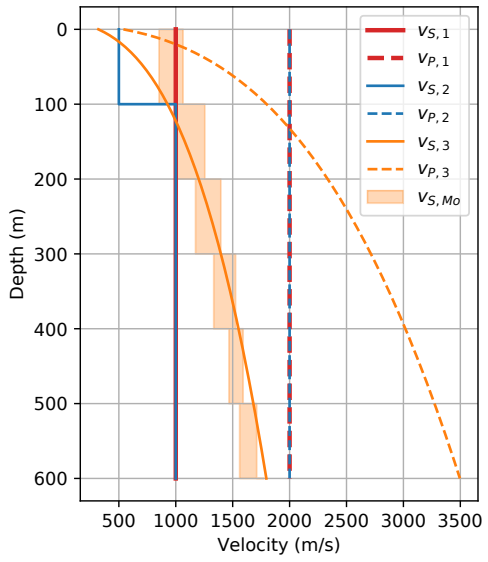

C)

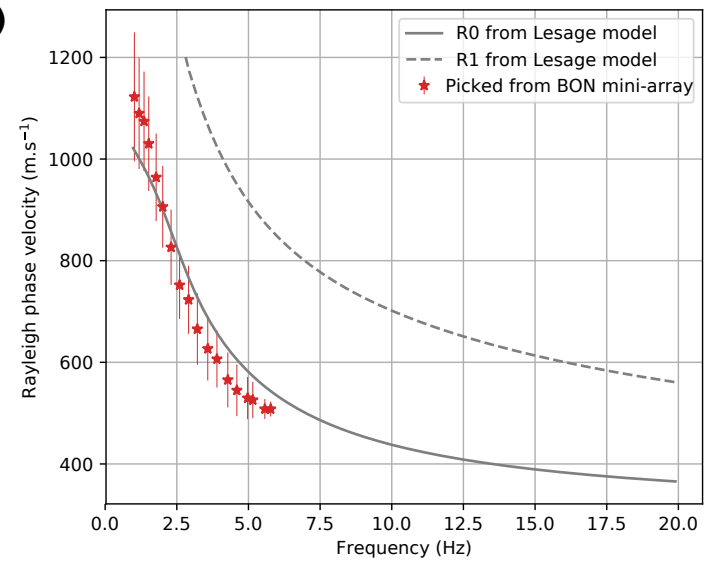

Figure 2. a) Cross-section of the SEM mesh through Dolomieu crater with a topography resolution of $10 \mathrm{~m}$. Perspective as seen from the East with Bory crater located in the background. The color map corresponds to the Lesage generic velocity model (see section 3.2). The buffer layer $100 \mathrm{~m}$ below the surface dampens small-scale topography variations. The zone of refinement at $150 \mathrm{~m}$ below the surface connects elements with $10 \mathrm{~m}$ and $30 \mathrm{~m}$ side lengths. $160 \mathrm{~m}$ wide PML boundaries are attached to the sides and bottom of the domain. b) S- and P-wave velocity depth profiles for the (1) homogeneous model $\left(v_{S, 1}\right.$ and $\left.v_{P, 1}\right),(2)$ model with shallow S-wave velocity layer $\left(v_{S, 2}\right.$ and $\left.v_{P, 2}\right)$, and (3) Lesage generic velocity model $\left(v_{S, 3}\right.$ and $\left.v_{P, 3}\right)$. The shaded zone $\left(v_{S, M o}\right)$ is extracted from the inverted 3D S-wave model of Mordret et al. (2015). c) Theoretical dispersion curves of the Lesage generic model for the fundamental (R0) and first-mode (R1) Rayleigh wave velocity together with picked dispersion curves from a mini-array around station BON. The errors are estimated from the uncertainty during dispersion curve picking.

plemented in Geopsy software (www.geopsy.org). Theoretical dispersion curves are calculated from the Lesage generic model using modal summation from Computer Programs in Seismology (Herrmann, 2013). The measured values agree well with the fundamental Rayleigh velocity dispersion curve. No coherent dispersion curves could be picked above $6 \mathrm{~Hz}$ because of the minimum mini-array aperture of $30 \mathrm{~m}$.

Despite missing measurements above $6 \mathrm{~Hz}$, the Lesage generic model is assumed to be the most reasonable model for the shallow high-frequency velocity structure of Piton de la Fournaise volcano because it is based upon measurements at comparable volcanoes. 
Table 1. Parameters of velocity models for the SEM simulations ${ }^{a}$

\begin{tabular}{|c|c|c|c|c|c|}
\hline Velocity model & $v_{P}$ & $v_{S}$ & $\rho\left(\mathrm{kg} \mathrm{m}^{-3}\right)$ & $Q_{P}$ & $Q_{S}$ \\
\hline 1) homogeneous & $2000 \mathrm{~m} \mathrm{~s}^{-1}$ & $1000 \mathrm{~m} \mathrm{~s}^{-1}$ & 2000 & 80 & 50 \\
\hline 2) low $v_{S}$ layer & $2000 \mathrm{~m} \mathrm{~s}^{-1}$ & $\begin{array}{l}500 \mathrm{~m} \mathrm{~s}^{-1}(\text { top } 100 \mathrm{~m}) \\
1000 \mathrm{~m} \mathrm{~s}^{-1}(\text { below } 100 \mathrm{~m})\end{array}$ & 2000 & 80 & 50 \\
\hline 3) generic & $\begin{array}{l}c_{P 0}=540 \mathrm{~ms}^{-1} \\
\alpha_{P}=0.315 \\
a_{P}=10\end{array}$ & $\begin{array}{l}c_{S 0}=320 \mathrm{~ms}^{-1} \\
\alpha_{S}=0.300 \\
a_{S}=15\end{array}$ & 2000 & 80 & 50 \\
\hline
\end{tabular}

${ }^{a} \mathrm{P}$ - and S-wave velocity $v_{P}$ and $v_{S}$, density $\rho$, and $\mathrm{P}$ - and $\mathrm{S}$-wave quality factor $Q_{P}$ and $Q_{S}$ for the (1) homogeneous model, (2) model with shallow S-wave velocity layer, and (3) Lesage generic velocity model.

The Lesage generic velocity model implemented on the SEM mesh is represented in Figure 2a. There are two options when implementing a velocity-depth profile on a 3D numerical domain with topography. The first possibility is to keep the velocity laterally homogeneous and excavate a surface corresponding to the topography. The second possibility is to adjust the velocity profile vertically so that it follows the topography elevation. Either way, the subsurface velocity structure is influenced, unless it is homogeneous. We chose the second option that we believe is geologically more reasonable because a main cause of velocity variation is the compaction of material with depth due to increasing overburden pressure.

Rock density $\rho$ as well as quality factors $Q_{P}$ and $Q_{S}$ for intrinsic attenuation of $\mathrm{P}$ - and S-wave velocity, respectively, are chosen based on previous studies on Piton de la Fournaise and similar volcanoes (Battaglia, 2003; O'Brien \& Bean, 2009; Hibert et al., 2011). All parameters are summarized in Table 1.

This work focuses on the topography effect, but it is important to have an idea of the effect of 3D-medium heterogeneities. Difficulties arise in this respect as there is lack of knowledge on the distribution of heterogeneities that is hard to invert from seismograms alone (Imperatori \& Mai, 2013). Nevertheless, a first attempt to simulate scattering effects is made by adding a spatially random velocity perturbation to the Lesage generic velocity model. The magnitude of the velocity deviation reaches $43 \%$ and is defined by a normal distribution with a standard deviation of $10 \%$, see Supporting Information.

\subsection{Topography resolution}

The influence of topography resolution on the simulated wave field is investigated to study influences from sub-wavelength topography variations and assess the trade-off between increased resolution and computation costs. Synthetic seismograms of the vertical component are compared (Figure 3a), obtained from models with a flat surface and topography resolutions of $20 \mathrm{~m}$ and $10 \mathrm{~m}$. A vertical point force in the form of $7 \mathrm{~Hz}$ Ricker source-time function is placed on the southern crater wall, corresponding to rockfall starting position R1 in Figure 1b.

The single-impact source produces a long wave-train of body waves and multiplemode Rayleigh waves. The maximum amplitudes decrease for the models with topography compared to the simulations with the flat model. The large-scale crater topography can redirect the wave-field and cause shadow-zones, as shown in section 4 where the spatial distribution of amplification or deamplification along the surface will be analyzed. Furthermore, topography causes prolonged and more complex waveforms. For the flat 
a)

BON

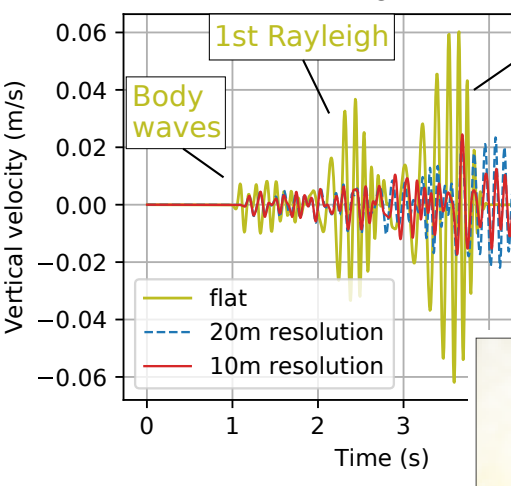

SNE

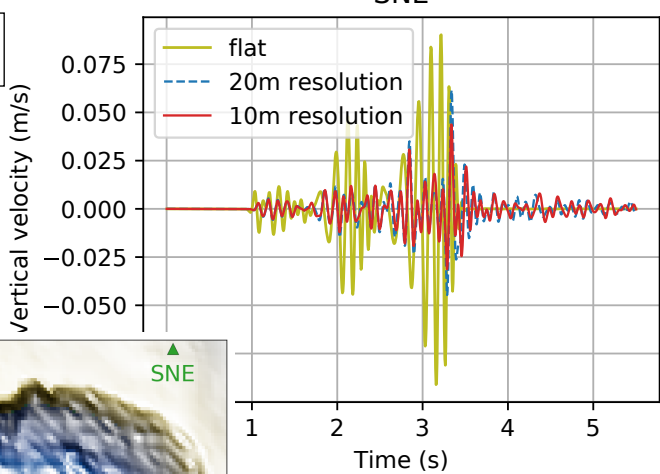
BOR
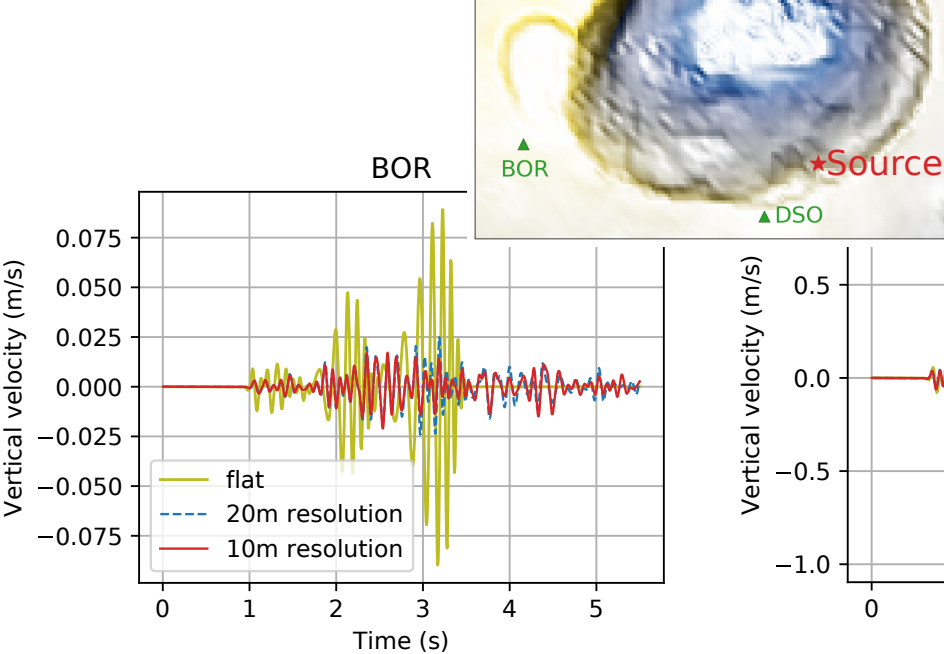

DSO

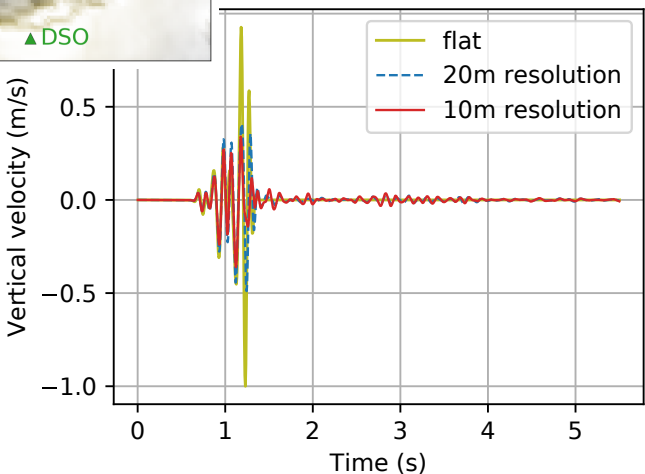

b)

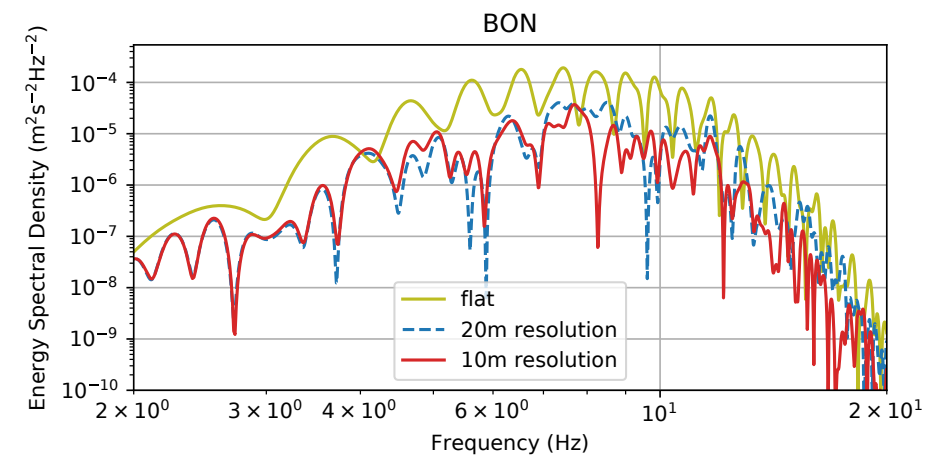

Figure 3. Influence of topography resolution on synthetic seismograms from the Lesage generic velocity model, recorded at stations BON, BOR, DSO and SNE. a) Comparison of synthetic seismograms (vertical velocity, normalized by maximum amplitude at closest station DSO) from the model with a flat surface, model with $20 \mathrm{~m}$ topography resolution (low-pass filtered with $30 \mathrm{~m}$ corner wavelength), and model with $10 \mathrm{~m}$ topography resolution. Seismograms recorded at stations BON, BOR, DSO, and SNE, surrounding Dolomieu crater. SEM configurations correspond to 7, 8, and 16 in Table A1. b) Corresponding spectra recorded at station BON.

model, wave packets corresponding to body waves, first-mode Rayleigh waves and fundamentalmode Rayleigh waves are well separated, but become less distinguishable when introducing topography. Comparing the two models with topography, note that the first part of the wave-train is almost identical, which is related to body waves not being affected by 
topography variations because of less interaction with the surface. Greater amplitude differences are found at later arrival times, suggesting that mainly slower surface waves of smaller wavelengths are affected by topography variations.

This assumption is supported by the spectra recorded at station BON, Figure 3b. Differences between the two models with topography become evident above roughly $5 \mathrm{~Hz}$. This corresponds to a minimum wavelength of $116 \mathrm{~m}$ for the fundamental Rayleigh wave $\left(\lambda \approx 580 \mathrm{~m} \mathrm{~s}^{-1} \div 5 \mathrm{~Hz} \approx 116 \mathrm{~m}\right)$. If we conclude that wavelengths below $116 \mathrm{~m}$ are still sensitive to the change in topography resolution, then $1^{\text {st }}$-mode Rayleigh waves of above $7 \mathrm{~Hz}$ are affected $\left(\lambda \approx 800 \mathrm{~m} \mathrm{~s}^{-1} \div 7 \mathrm{~Hz} \approx 114 \mathrm{~m}\right)$. This analysis suggests surface waves are sensitive to changes in topography resolution that are 5 times smaller than their wavelength.

The decrease in amplitude at all stations for the high-resolution topography $(10 \mathrm{~m})$ relative to the lower-resolution topography $(20 \mathrm{~m})$ suggests that more energy is scattered during the propagation of the surface waves and possibly lost within the subsurface. Interestingly, S. J. Lee, Chan, et al. (2009) found the opposite when comparing waveforms on different topography resolutions for a source deep below the surface. This implies that the source position plays a major role in the effect of topography. On one hand, topography can increase ground shaking and thus trap energy close to the surface. On the other hand, in the case of waves traveling along the surface, the topography can increase scattering and thus prevent energy propagation. Similar conclusions were drawn by S. J. Lee, Komatitsch, et al. (2009), who investigated how topography effects are modulated by the source depth in regard to ground motion in a basin located behind a mountain range.

\subsection{Wave propagation from a vertical surface load}

To better understand wave propagation along the topography and the influence of the subsurface geology, snapshots of the wave field were examined. Simulations for all three velocity models were carried out on the domain with $20 \mathrm{~m}$ Dolomieu topography resolution and a vertical point force on the southern crater wall.

Figure 4 shows synthetic seismograms recorded on the surface along an array crossing the source position, Dolomieu crater and station BON (see inset for location of the array). Snapshots of the propagating seismic wave field on a cross-section along the array are shown below the seismograms. All amplitudes correspond to vertical ground velocity. In order to enhance visibility of the wave field over time, the simulations here are carried out without intrinsic attenuation.

For the simulation with the homogeneous domain (left column of Fig. 4), we can identify in the first snapshot at time $t=0.8 \mathrm{~s}$ the P-wave traveling downwards as being the fastest wave with propagation direction parallel to the shown vertical ground velocity. At time $t=1.6 \mathrm{~s}$, the original S-wave is visible on the bottom of the cross-section. The S-wave can be identified because the direction of propagation is perpendicular to the vertical ground velocity. Just above, note the newly created S-wave (annotated as RS) that separated at the bottom of the crater from the Rayleigh wave because of the convex topography. Yet, part of the energy continues as a Rayleigh wave along the topography towards the rim of the crater. Also visible is a diffracted surface wave (annotated as Rd). It split from a wave front traveling towards station BOR and took a curved path along the flank of the crater. At time $t=2.0 \mathrm{~s}$ we can see this diffracted Rayleigh wave continuing outside the crater and arriving at station BON at a different azimuth than that of the Rayleigh wave that traveled diagonally across the crater and its rim (annotated as Rf). The energy of Rayleigh wave $\mathrm{Rf}$ was partly reflected at the crater rim so that a new Rayleigh wave Rr traveled backwards through the crater. Up front (on the far right of the domain), a direct S-wave hits the surface and is partly reflected and converted to build a straight $\mathrm{P}$-wave front traveling downwards at an oblique angle to the horizontal (annotated as SP).

Adding a low S-wave velocity layer (middle column in Fig. 4) drastically changes the wave field because of reflections within this layer and the dispersive character of Rayleigh 


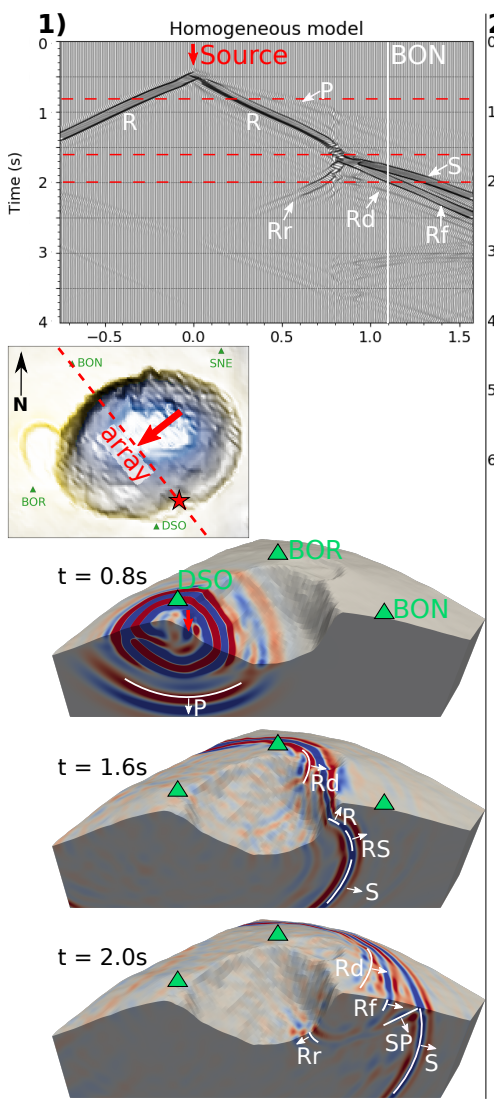

2) Low velocity layer model

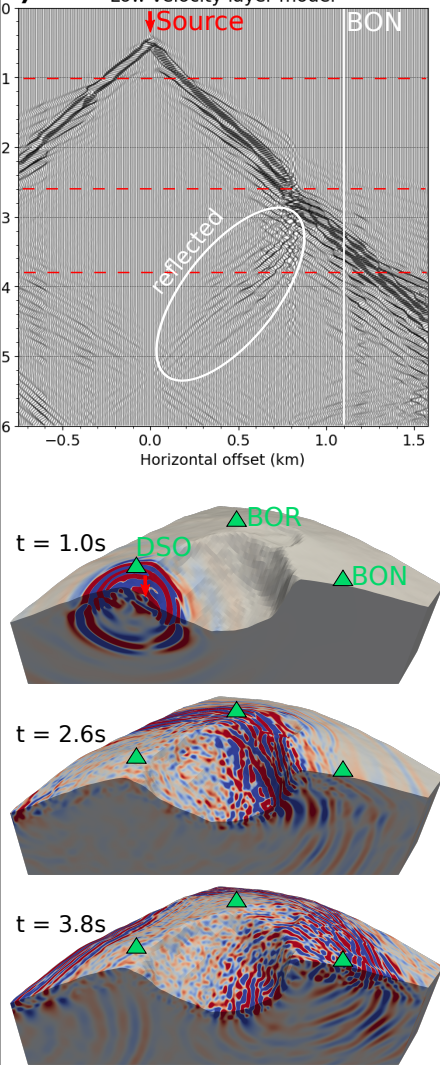

3)
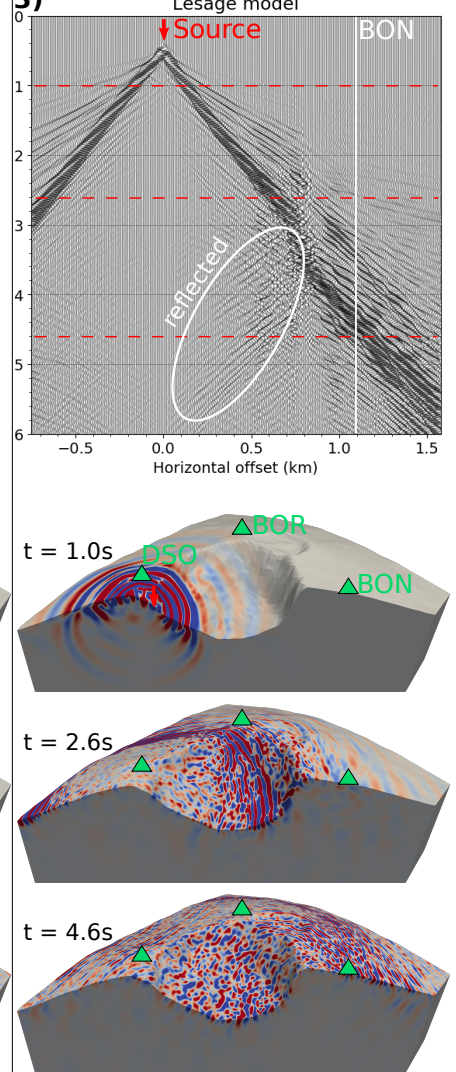

Figure 4. Wave propagation from a vertical surface load for different velocity models. Synthetic seismograms (top row) recorded at an array crossing the source, Dolomieu crater and station BON (see inset) for the (1) homogeneous model (left), (2) model with a shallow S-wave velocity layer (middle), and (3) Lesage generic velocity model (right). The seismic traces are normalized with respect to themselves and show vertical ground velocity. Snapshots of the wave field on cross-sections along the same array are shown below the seismograms, corresponding to the times marked by red dashed lines. Annotations denote $\mathrm{P}$-wave $(\mathrm{P})$, S-wave (S), P to S converted wave (PS), Rayleigh to S converted wave (RS), Rayleigh wave (R), reflected Rayleigh wave (Rr), diffracted Rayleigh wave (Rd), and diagonally traveled Rayleigh wave (Rf). SEM configurations correspond to 3, 6 and 11 in Table A1. The absence of intrinsic attenuation for enhanced visibility of wave propagation caused reflections from the boundary on the left at later times.

waves. Looking at the first $2.5 \mathrm{~s}$ of the synthetic seismograms, we observe a wave-train with a dispersive character overlaid by multiples. Compared to the homogeneous model, it is more complex and has a longer duration because of internal reflections within the low-velocity layer. At around $t=2.6 \mathrm{~s}$, the waves hit the crater rim opposite the source and are partly reflected just like in the homogeneous case. The snapshots at times $t=$ $2.6 \mathrm{~s}$ and $t=3.8 \mathrm{~s}$ show, in contrast to the homogeneous case, a much more scattered wave field of irregular amplitude patterns. Similar to S. J. Lee, Chan, et al. (2009), who found characteristic patterns dependent on the resolution of the imposed topography, the characteristic length of these patterns is likely to be related to the resolution of the topography and the flat element surfaces with $20 \mathrm{~m}$ side lengths.

For the Lesage generic velocity model (right column of Fig. 4), the majority of energy stays close to the surface of the domain because of the velocity gradient. Scatter- 
ing of the wave field over the topography is even greater than for the case with a lowvelocity layer and the duration of shaking is increased. From synthetic seismograms (top right of Fig. 4), we can still identify the outward propagation of energy as well as the reflection of part of the energy at the crater rim. The analysis of the simulations shows that a single impact can produce a complex wave field caused by the surface topography and the underlying velocity model.

Regarding scattering from 3D medium heterogeneities, results from simulations in which the Lesage velocity model is randomly perturbed by heterogeneities with a standard deviation of $10 \%$ (with maximum excess of $43 \%$ ) show barely affected synthetic signals, see Supporting Information, indicating that the effective medium is not changed significantly. This example does not prove that scattering is weak as it is possible to design a distribution of characteristic correlation lengths that completely changes the waveforms. However, studies at Dolomieu crater lead us to believe that this is not the case; e.g. Hibert et al. (2011) found that rockfall seismic signals do not exhibit a coda but that their duration corresponds to the rockfall propagation time on videos and Kuehnert et al. (2020) tracked rockfall trajectories using simulated inter-station energy ratios with the smooth Lesage velocity model.

\section{Influence of topography on simulated wave propagation}

Seismic amplitudes carry crucial information on the seismic source and can be used to infer source locations and acting forces. However, as can be concluded from the simulated wave propagation above, topography together with the underlying geology can strongly influence ground motion. Consequently, the measured amplitudes have to be interpreted according to both source properties (including the resulting radiation patterns) and propagation effects. In the following, topography induced amplification is quantified for different velocity models and different source directions. This can be helpful to better interpret the spatial distribution of amplitudes and eventually account for amplified signals.

\subsection{Amplification for a vertical source}

In order to quantify topographic ground motion amplification, simulations on a model with topography are compared to a reference model with a flat surface. The comparison is performed for both vertical peak ground velocity $\mathrm{PGV}_{z}$ and total kinetic energy E. Quantifying PGV amplification is important when interpreting signal amplitudes. However, it does not measure the increased complexity and duration of recorded waveforms caused by scattering and diffraction of the wave field along the topography. These effects can be incorporated by calculating energy amplification. Also, frequency dependencies are not considered. For this reason we will later look at different frequency bands or determine spectral ratios when analyzing observed rockfall signals.

To quantify vertical PGV amplification, the maximum vertical ground velocity is measured at each point on the surface defined on a grid with $30 \mathrm{~m}$ spacing. The top row of Figure 5 shows the peak ground velocity ratios $\mathrm{PGV}_{z, T} / \mathrm{PGV}_{z, F}$ between the three velocity models with topography and the flat reference model.

Similarly, energy amplification is calculated at each grid point by the ratio $E_{T} / E_{F}$ between the models with topography and the flat reference model, where $E_{i}$ (with $i=$ $T, F)$ is a proxy of the total kinetic-energy density, defined as the square of the recorded ground velocity $\vec{v}$, integrated over the total signal duration $d$ :

$$
E_{i}=\int_{d}\left(v_{x, i}^{2}(t)+v_{y, i}^{2}(t)+v_{z, i}^{2}(t)\right) \mathrm{d} t
$$

The resulting energy amplification is shown in the bottom row of Figure 5 for the three different velocity models. 

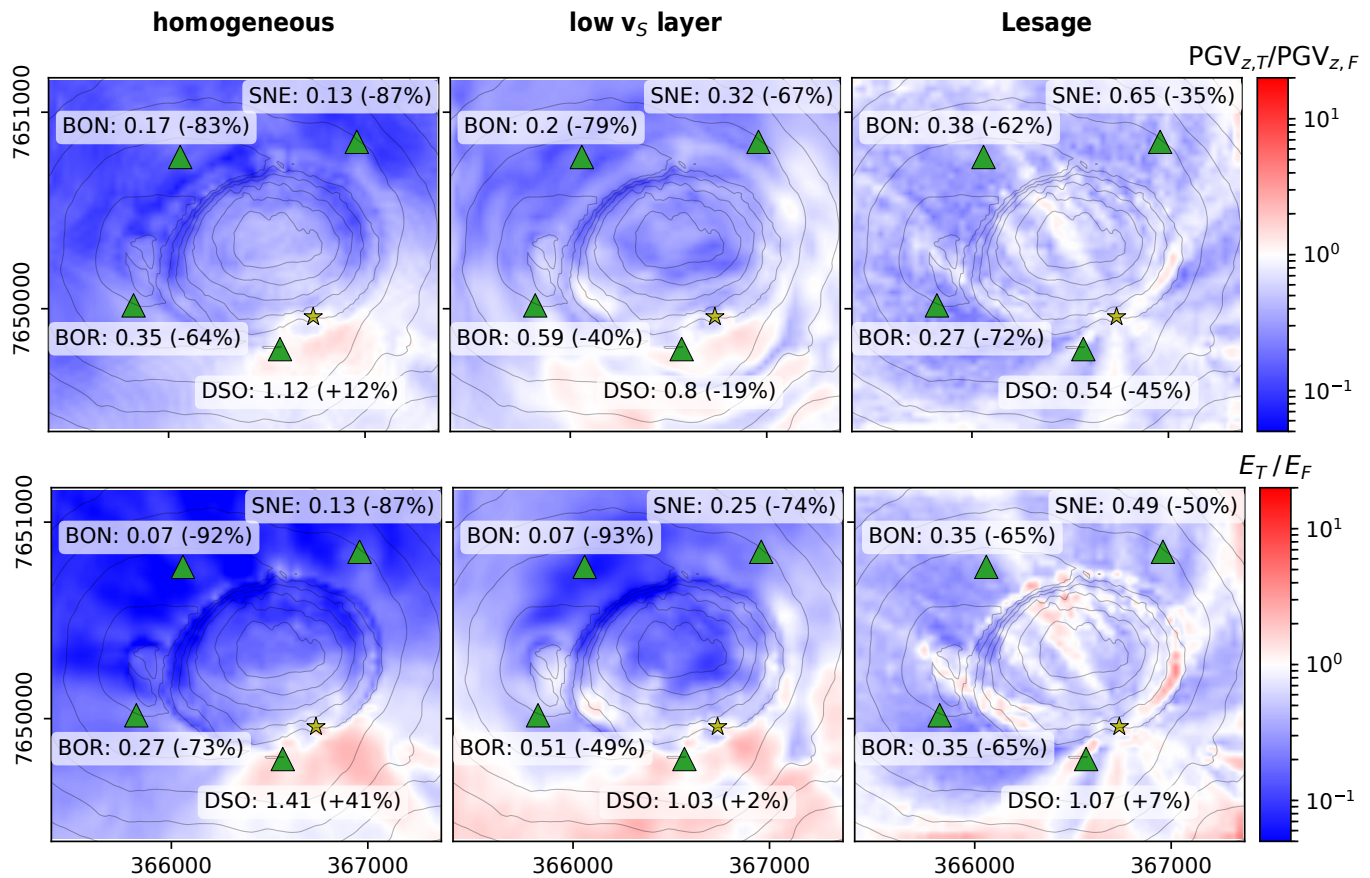

Figure 5. Topographic amplification from for a vertical point force. Amplification for of vertical PGV (top) and total kinetic energy (bottom) is calculated relative to a flat reference model for the homogeneous model (left), the model with a shallow low velocitylow-velocity layer (middle) and the Lesage generic velocity model (right). SEM configurations correspond to 1, 2; 4, 5;

7, 8 in Table A1. The yellow star illustrates the source position and green triangles mark station locations. Annotations give ratios measured at the station locations as well as the percentage of topographic amplification. Neighboring contour lines differ by $60 \mathrm{~m}$ in elevation.

\subsubsection{PGV amplification}

Analyzing the PGV amplification shown in the top row of Figure 5, the homogeneous model shows a contrast between source side of the crater and the opposite side: PGV is amplified on the source side and strongly deamplified on the far side. The amplification on the source side $(+12 \%$ at DSO) can be explained by the simultaneous arrival of surface and direct waves emitted from the source. Deamplification on the farside of the source $(-83 \%$ at $\mathrm{BON}$ and $-87 \%$ at $\mathrm{SNE})$ can be interpreted as a shadow zone behind the crater related to the diversion of a major part of wave energy downwards into the subsurface because of the crater shape.

For the model with the low-velocity layer, general amplification on the source side and deamplification on the far-source side of the crater are still present but less pronounced (deamplification at station SNE is reduced to -67\%) and patterns become more complex (DSO is now deamplified by $-19 \%$ ). The introduction of a low-velocity layer causes more energy to stay at the surface and thus reduces the shadow zone behind the crater. The uneven topography together with the underlying low-velocity layer causes complicated reflections and wave conversions which lead to increased complexity of amplification patterns.

The contrast between source side and far-source side of the crater decreases further for the Lesage generic velocity model $(-45 \%$ at $\mathrm{DSO},-62 \%$ at $\mathrm{BON}$ and $-35 \%$ at $\mathrm{SNE}$ ). As can be seen on the wave propagation snapshots in Figure 4, the gradient causes energy to stay close to the surface. Whereas a lot of energy is lost downwards because 
of the crater topography in the homogeneous model as well as in the low-velocity layer model, the velocity gradient in the Lesage generic model guides waves efficiently along the crater topography or back to the surface, which causes a more homogeneous amplification pattern. Scattering away from the surface due to surface roughness as well as conversion from vertical to horizontal energy leads to an overall deamplification in vertical PGV. Still, because of focusing mechanisms of the 3D topography, ray-shaped zones of PGV amplification originating at the source can be observed.

\subsubsection{Energy amplification}

In general, the amplification patterns of kinetic energy (bottom row of Figure 5) show more contrast than the PGV ratios. This is because topography does not only influence peak amplitude, but also the complexity and duration of the signal. For the homogeneous model, amplification increases to $+41 \%$ at DSO and decreases to $-92 \%$ at BON. Behavior for the model with the low-velocity layer is very similar. For the Lesage generic model, the ray-shaped zones of amplification are considerably more pronounced than for the case of PGV amplification. Given that horizontal ground velocity is considered when computing the kinetic energy, this observation suggests that topography guides both vertical and horizontal energy along the same paths. Note also the increased amplification at parts of the crater cliff ridge, possibly due to the discussed reflection of Rayleigh waves at these positions.

Changing the velocity model modifies the wavelengths, which presents an alternative explanation for the observed differences in the amplification patterns. This explanation was discarded after verifying that the differences still remain for bandpass filtered results, comparing amplification patterns from the different velocity models for coinciding wavelengths as done in Appendix B.

\subsection{Amplification for a horizontal source}

Only vertical surface loads are considered above. However, the rockfall generated basal forces on the ground can also have horizontal components. Here we show amplification patterns for a horizontal source using the Lesage generic velocity model. Figure 6 illustrates vertical PGV amplification (left) and energy amplification (right) for a wave field generated by a horizontal surface force polarized in the north direction.

A strong directionality is visible in the PGV amplification pattern. This is because for the flat reference model, a horizontal source does not generate vertical seismic energy perpendicular to its direction. Topography however can change this by conversion from transverse energy or diffraction of wave paths.

The directionality patterns are no longer visible when analyzing the amplification of total kinetic energy. This is because all components of the measured ground velocity are considered in the energy calculation. The energy amplification pattern is similar to the one for the vertical source as shown above in Figure 5. This suggests that topography guides seismic energy on trajectories along the surface that mainly depend on the source position rather than the source direction. We will further discuss this hypothesis later when studying inter-station spectral ratios of real rockfall signals.

\subsection{Surface roughness and crater geometry}

The amplification patterns observed in the previous section are characterized by complex spatial distributions. We will now perform tests on domains with synthesized surface topographies in order to better understand the contributions of certain geometric features to the amplification pattern. More concretely, we will study a planar surface with natural roughness as well as synthetic crater shapes of different depths and curvatures. Surface roughness and crater dimensions are defined to resemble our study site on Piton de la Fournaise volcano. The initial domain is a cube of size $2360 \mathrm{~m} \times 2360 \mathrm{~m} \times$ 

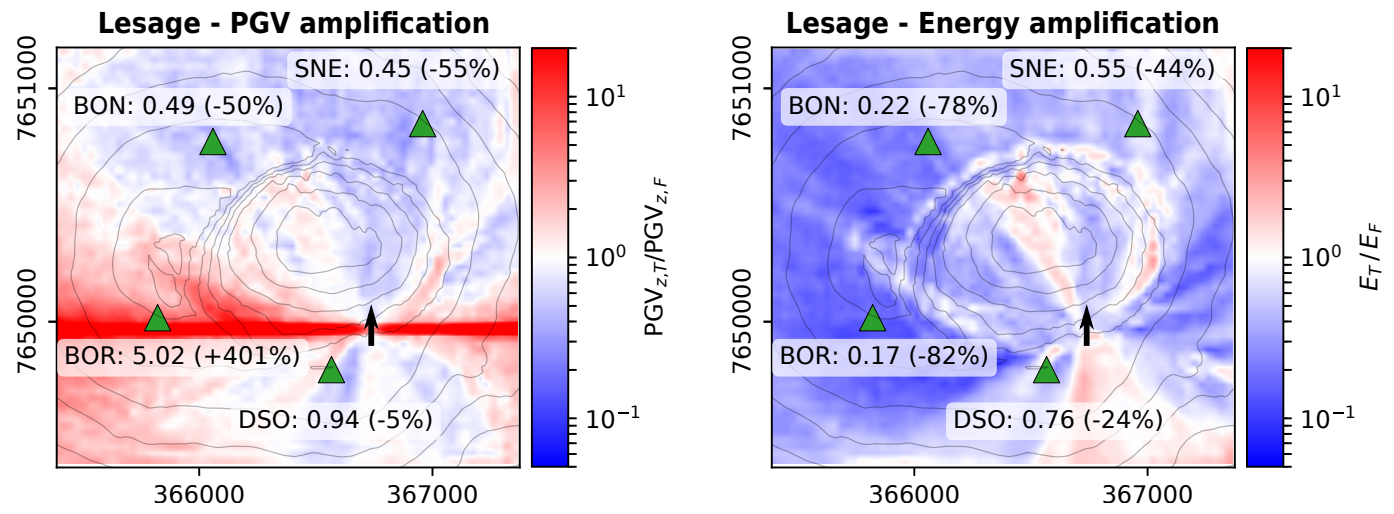

Figure 6. Topographic amplification for a horizontal point force in the north-direction. Amplification of vertical PGV (left) and total kinetic energy (right) is calculated relative to a flat reference model for the model with the Lesage generic velocity profile. SEM configurations correspond to 9 and 10 in Table A1. The black arrow illustrates the source position and its direction. Green triangles mark station locations. Annotations give ratios measured at the station locations as well as the percentage of topographic amplification. Neighboring contour lines differ by $60 \mathrm{~m}$ in elevation.

$600 \mathrm{~m}$, meshed by elements with $20 \mathrm{~m}$ side lengths. The subsurface medium of all domains corresponds to the Lesage generic velocity model. As above, a $7 \mathrm{~Hz}$ Ricker wavelet is used as the surface point force.

The domain with a planar rough surface is constructed from an area of the DEM at Piton de la Fournaise volcano and band-pass filtered at corner wavelengths of $40 \mathrm{~m}$ and $100 \mathrm{~m}$. In this way, minimum and maximum wavelengths of the fundamental Rayleigh wave in the Lesage generic model are below and above the range of topography wavelengths, respectively (i.e. $\lambda_{15 \mathrm{~Hz}} \approx 390 \mathrm{~m} \mathrm{~s}^{-1} \div 15 \mathrm{~Hz}=26 \mathrm{~m}$ and $\lambda_{5 \mathrm{~Hz}} \approx 580 \mathrm{~m} \mathrm{~s}^{-1} \div$ $5 \mathrm{~Hz}=116 \mathrm{~m}$ ). To design a typical crater geometry, we use the equation proposed by Soontiens et al. (2013). However, using a smooth, symmetric crater shape results in symmetric interferences. To avoid artificial amplification patterns of perfect symmetry, the above defined surface roughness is added to the elevation values of the synthetic crater shape. The corresponding SEM meshes are shown in the Supplementary Information.

Figure 7a compares synthetic seismograms recorded along arrays on the domains with a flat surface, a planar rough surface, and crater topography.

For the model with the flat domain, we can identify dispersive fundamental and first-mode Rayleigh waves as well as body waves. Introducing surface roughness leads to strong scattering and hence prolonged ground shaking. The two Rayleigh modes are no longer clearly separated, even though the propagation of the main energy from the fundamental mode can be identified. Introducing the crater topography adds more complexity. In particular the steep crater walls distort the propagating wave field, as already observed for the real crater topography (see Fig. 4).

We now investigate the effect of surface topography at different frequency bands. For this, we quantify as before the amplification of total kinetic energy with respect to the flat reference model. Note that here we present energy instead of PGV as it accounts for both amplitudes and prolonged ground shaking and hence gives a more general picture. Figure $7 \mathrm{~b}$ shows energy amplification on both the rough planar domain and the domain with a synthetic crater in the $3-7 \mathrm{~Hz}$ and $13-17 \mathrm{~Hz}$ frequency bands.

We can see that both these frequency bands are influenced by the rough planar surface. As already indicated, the rough topography is band-pass filtered at corner wavelengths $40 \mathrm{~m}$ and $100 \mathrm{~m}$ and the minimum and maximum wavelengths of fundamental Rayleigh waves are below and above the range of topography wavelengths, respectively. 
a)
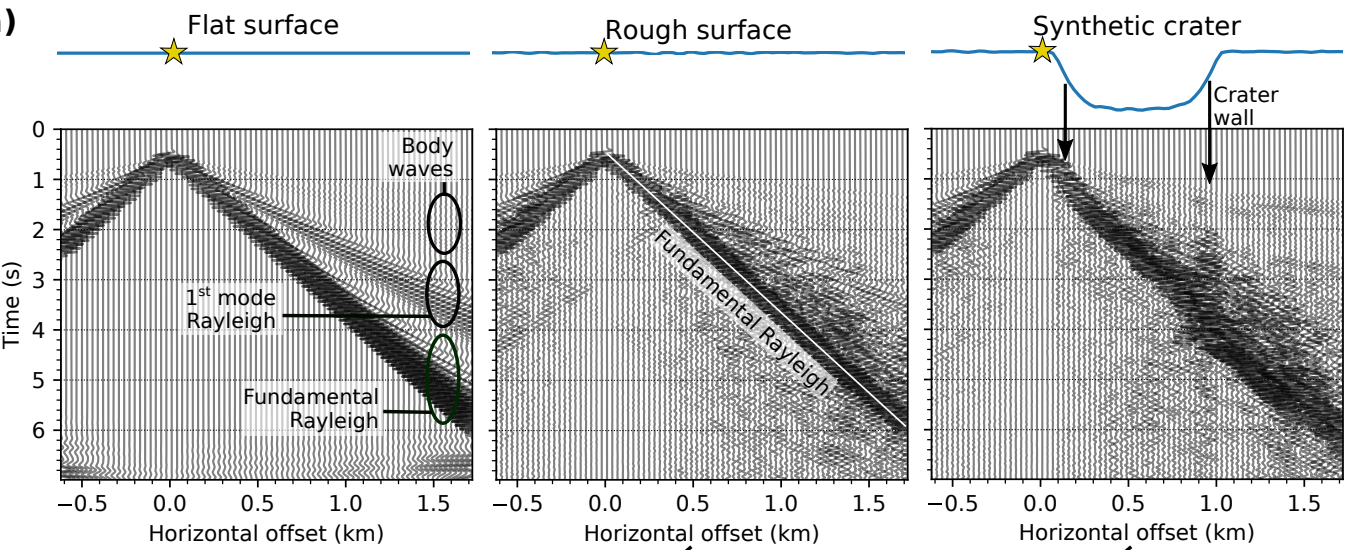

b)

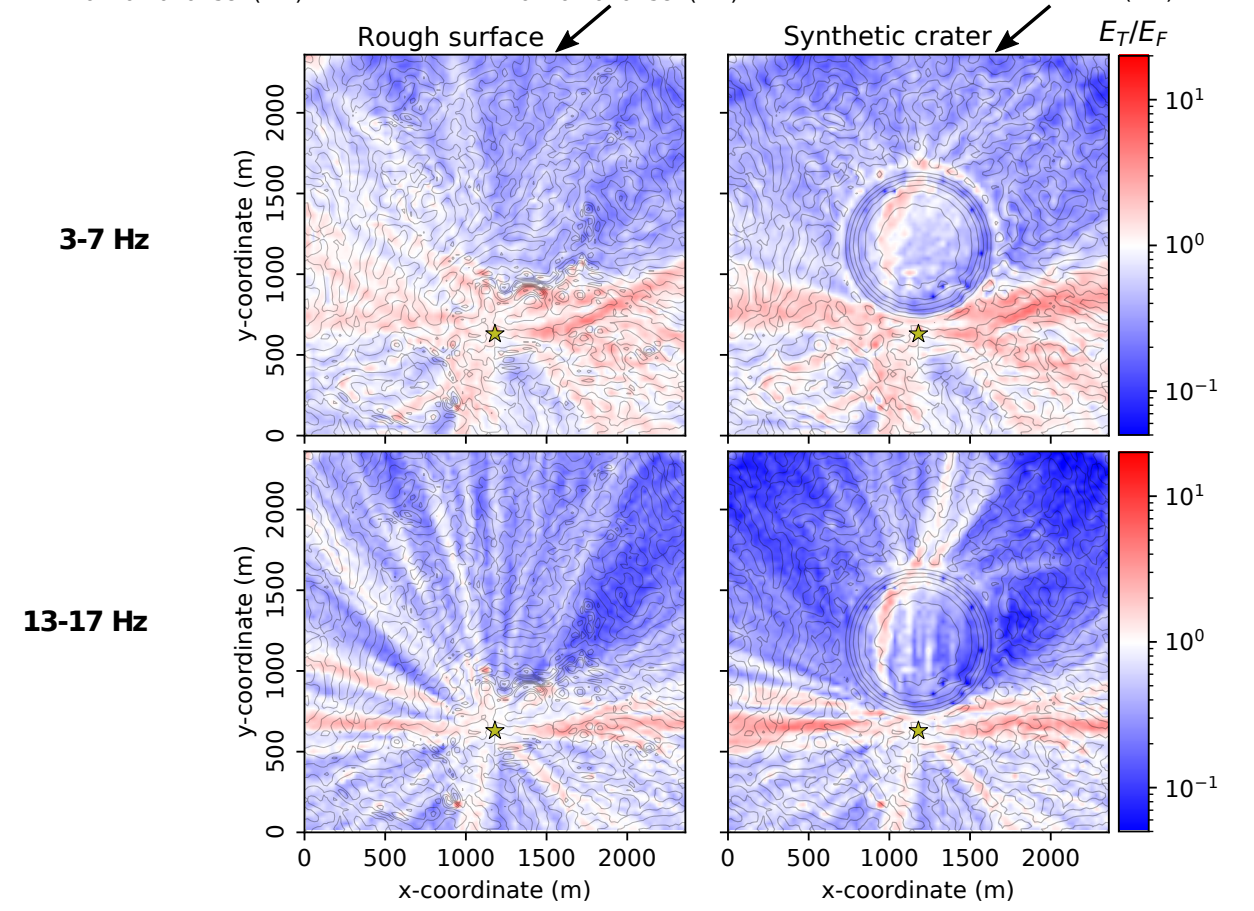

Figure 7. a) Synthetic seismograms of vertical ground velocity from models with a flat surface (left), a rough planar surface (middle), and a synthetic crater shape (right). Seismograms are normalized and recorded along the surface profiles illustrated by the blue curves on top. The yellow star marks the position of the vertical source. The spurious reverberations observed for the flat surface model after the signal $(>6 \mathrm{~s}$ ) are trimmed for the analyses. b) Energy amplification in different frequency bands for the model with a rough surface (left) and with a synthetic crater (right) relative to the flat reference model for frequency bands $3-7 \mathrm{~Hz}$ and $13-17 \mathrm{~Hz}$. The arc-like features at $3-7 \mathrm{~Hz}$ in the top corners are numerical artefacts. SEM configurations correspond to 12,13 and 14 in Table A1.

We observe ray-shaped zones of amplification which are blurred in the lower-frequency band and become sharper towards higher frequencies, because of the shorter interfering wavelengths. The variations of topography seem to guide energy along these ray paths. In contrast, some areas of pronounced topography variation (visible by the densification of contour lines) seem to shield the propagation of energy and cause shadow zones behind them. This can for example be observed in the north-east direction of the source. 


\section{a) Varying depth}
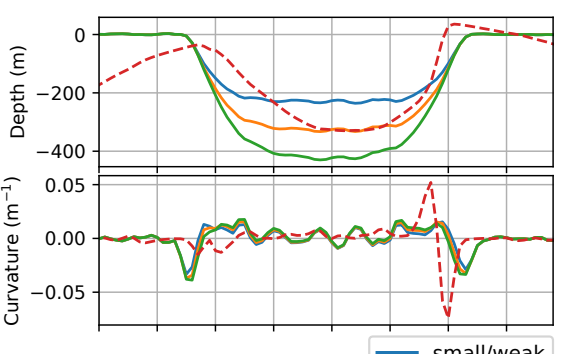
small/weak
medium

b) Varying curvature

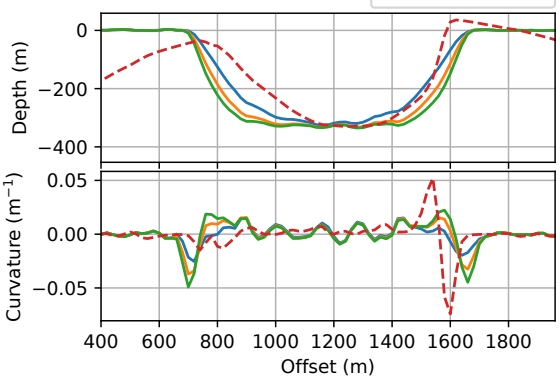

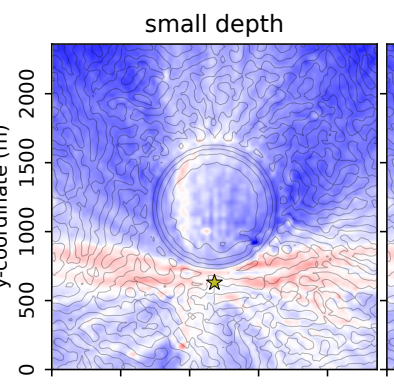

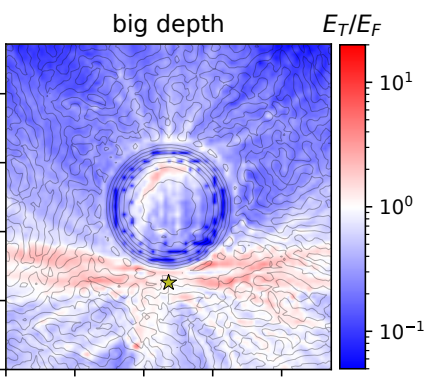

weak curvature

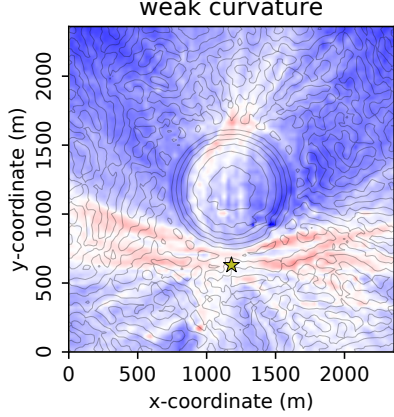

strong curvature $\quad E_{T} / E_{F}$

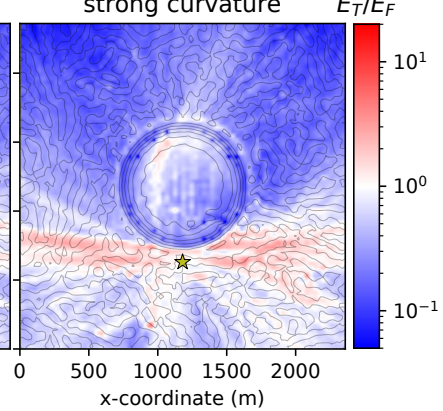

Figure 8. Left: Profiles through the synthetic crater topographies. a) Crater depths vary by \pm 0.3 from small to big with fixed curvature. b) Curvatures vary by \pm 0.3 from weak to strong with fixed crater depth. Red dashed lines correspond to a profile through Dolomieu crater and its corresponding curvature. Right: Comparison between energy amplification for crater geometries with smallest and biggest depths as well as weakest and strongest curvature. Contour lines mark elevation differences of $50 \mathrm{~m}$ and the yellow star denotes the source. Note that spurious blue dots inside the crater (especially at steep flanks for the big depth) were caused by numerical measurement problems at these positions. SEM configurations correspond to 12 and 14 in Table A1.

The energy ratios from the simulations on the domains with synthetic crater shapes are shown on the right-hand side of Figure 8 for the whole frequency range. The amplification pattern varies slightly going from small depth to big depth (Figure 8a). The biggest change is observed behind the crater directly opposite the source. Amplification decreases 
at this point with increasing crater depth. In contrast, increased amplification is observed inside the crater. These changes in the amplification patterns might be related to interference caused by the symmetric crater form. Going from weak to strong curvature (Figure $8 \mathrm{~b}$ ), the shadow zone behind the crater increases more strongly. This is not only true directly opposite the source position but also diagonally across the crater, suggesting that the increased crater curvature shields off more energy by reflecting or deflecting the wave field sideways or downwards into the subsurface.

The analyses suggest that variations of curvature have stronger effects on ground motion than variations of crater depth for models tested. It is important to note that the wave field is influenced by topography features of scales both below and above the seismic wavelength. This was observed for the experiments with a planar rough surface as well as with the synthetic crater with dimensions $(\sim 800 \mathrm{~m}$ diameter, $\sim 300 \mathrm{~m}$ depth $)$ largely exceeding the seismic wavelengths.

The experiments on the synthetic model surfaces explored effects of individual aspects of the topography on the wave field. The insights acquired can be transferred to our study side at Piton de la Fournaise volcano as similar scales were chosen deliberately. Having said this, note that the overall effect on the wave field is governed by the whole configuration and cannot be reduced to an individual feature (e.g. only small-scale versus only big-scale topographic variations). At the same time, the relative position of the source and receiver plays a defining role, i.e. topography related amplitude modifications at a given station can only be predicted if the source position is known.

\section{Seismic signals from rockfalls at Dolomieu crater}

We will now study observed seismic signals generated by rockfalls at Dolomieu crater. As the influence of the topography changes with the source position, we analyze the signals at specific times corresponding to specific rockfall positions. First we will investigate spectral ratios between stations of time windowed rockfall signals. The objective is to clarify as to whether simulations can reproduce the observed spectral ratios when taking into account topography. Subsequently we will focus on a single block impact, identifying its seismic signature and comparing observed and simulated amplitudes by estimating the generated impact force using Hertz contact theory.

\subsection{Observed spectral ratios between stations}

For this analysis, we select three rockfalls with similar trajectories on the southern crater wall corresponding to rockfall location $\mathbf{1}$ in Figure 1b. The trajectories of the rockfalls were identified from camera recordings. Snapshots of the three events are shown in Figure 9 together with an image of the whole trajectory reconstructed from differences of successive snapshots. Below, the corresponding seismic signals recorded at stations BON, BOR, DSO and SNE are presented.

Station DSO shows the strongest amplitudes, especially in the beginning of the rockfall. This is because the three rockfalls start very close to this station. BON contains the smallest amplitudes, being the furthest station and on the opposite side of the crater. The dynamics of the three events are not entirely identical. Event 1 consists of a single boulder bouncing down towards the bottom of the crater while other blocks follow with a time lag of around $15 \mathrm{~s}$. In contrast, event 2 consists of two blocks closely following each other down with a time lag of only $4 \mathrm{~s}$, as can be seen on snapshot $2 \mathrm{~b}$. Event 3 consists of a main boulder with a smaller block following much later with a lag of about $50 \mathrm{~s}$.

Despite these differences, we compare spectral ratios between stations in time windows R1, R2 and R3 during which the main blocks moved within identical areas. The spectral ratios are computed from the measurements at stations BOR, DSO (vertical component only) and SNE with respect to station BON (note that BON is selected as the reference station as it turns out it is the least affected by local site effects). In order to avoid spurious fluctuations, the spectra are smoothed as proposed by Konno and Ohmachi 

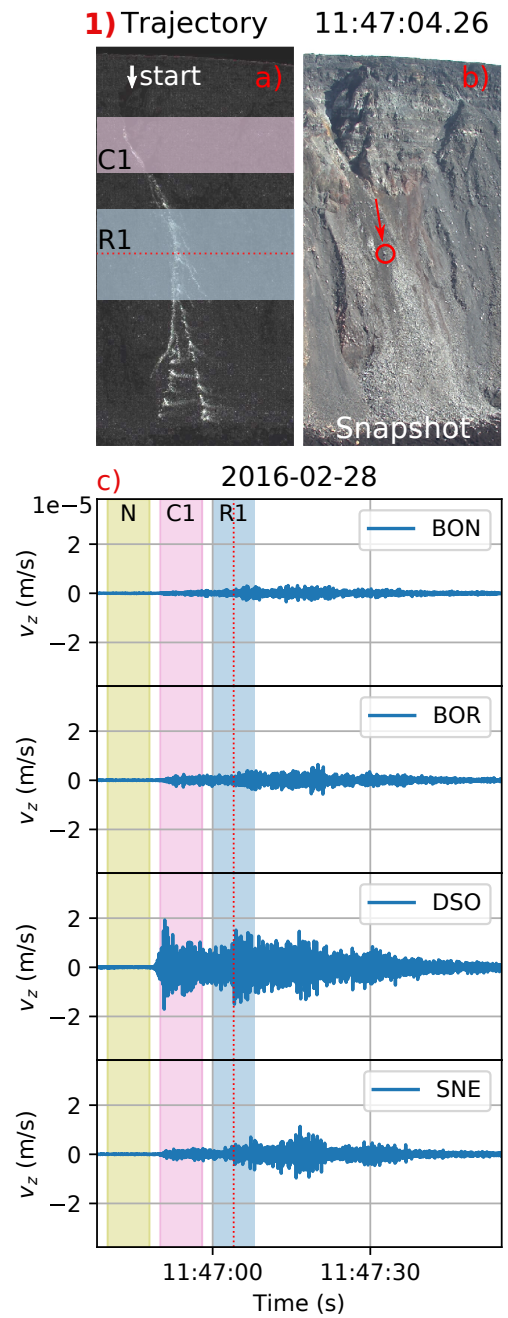

11:47:04.26 2)Trajectory
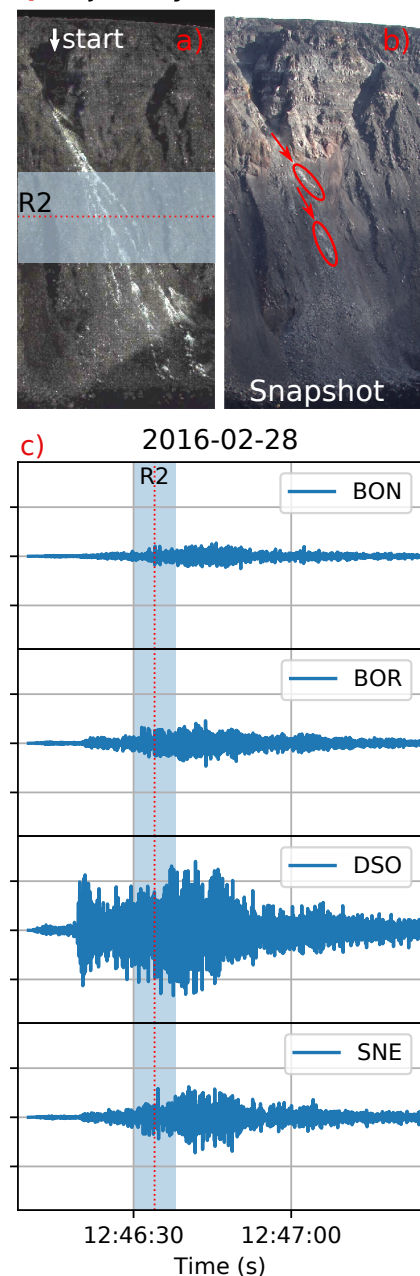

$12: 27: 38.23$
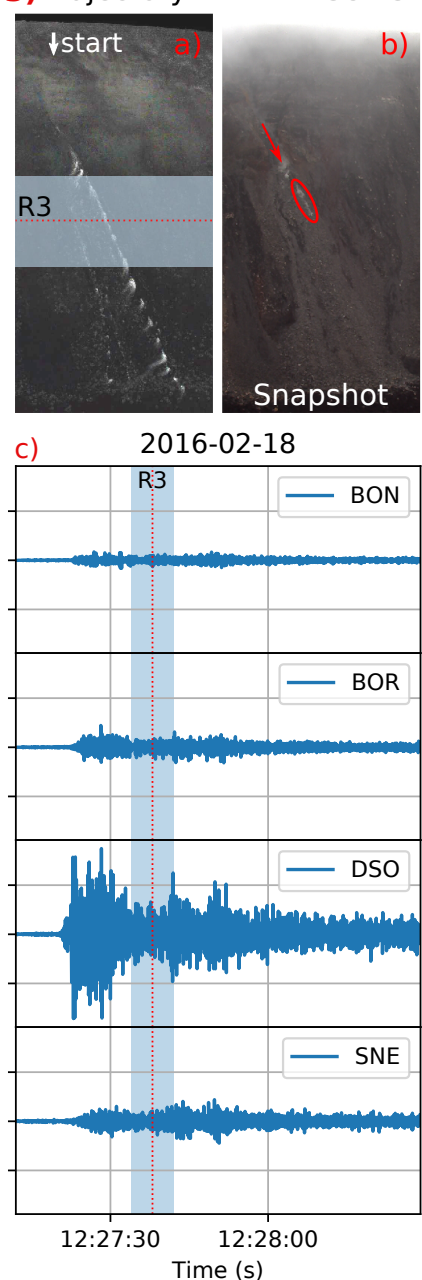

Figure 9. Three similar rockfalls on the southern wall of Dolomieu crater, corresponding to rockfall location 1 in Figure 1b. The events occurred on: 1) February 28, 2016 at around 11:47, 2) February 28, 2016 at around 12:46, and 3) February 18, 2016 at around 12:27. a) The total trajectory of each event (seen from camera SFRC). The approximate starting positions at the top of the crater wall are indicated by white arrows. b) Snapshots (seen from camera SFRC) at a chosen time at which all three rockfalls are at comparable positions. c) The rockfall seismic signals, the red dotted lines indicating the times of the snapshots. Time windows R1, R2, and R3 (blue-shaded zones) are defined $\pm 4 \mathrm{~s}$ around these times. The corresponding locations of these time windows are also indicated as blue-shaded zones on the trajectories. The same holds for reference time window C1 (magenta-shaded zone), which corresponds to the beginning of event 1 . Noise time window $\mathrm{N}$ is taken from recordings before event 1.

(1998) before calculating the ratios. The obtained curves are shown as dark blue lines (TW-R1, -R2, -R3) in Figure 10 for vertical- (top), north- (middle) and east- (bottom) components. Note the similar behavior of the spectral ratios for each of the events and for each component.

Comparison to spectral ratios from noise recordings (TW-N), from the beginning of event 1 (TW-C1), and from a rockfall that occurred at a different position in the crater (TW-C2, corresponding to trajectory $\mathbf{2}$ in Figure 1b), shows partly strong deviations from 


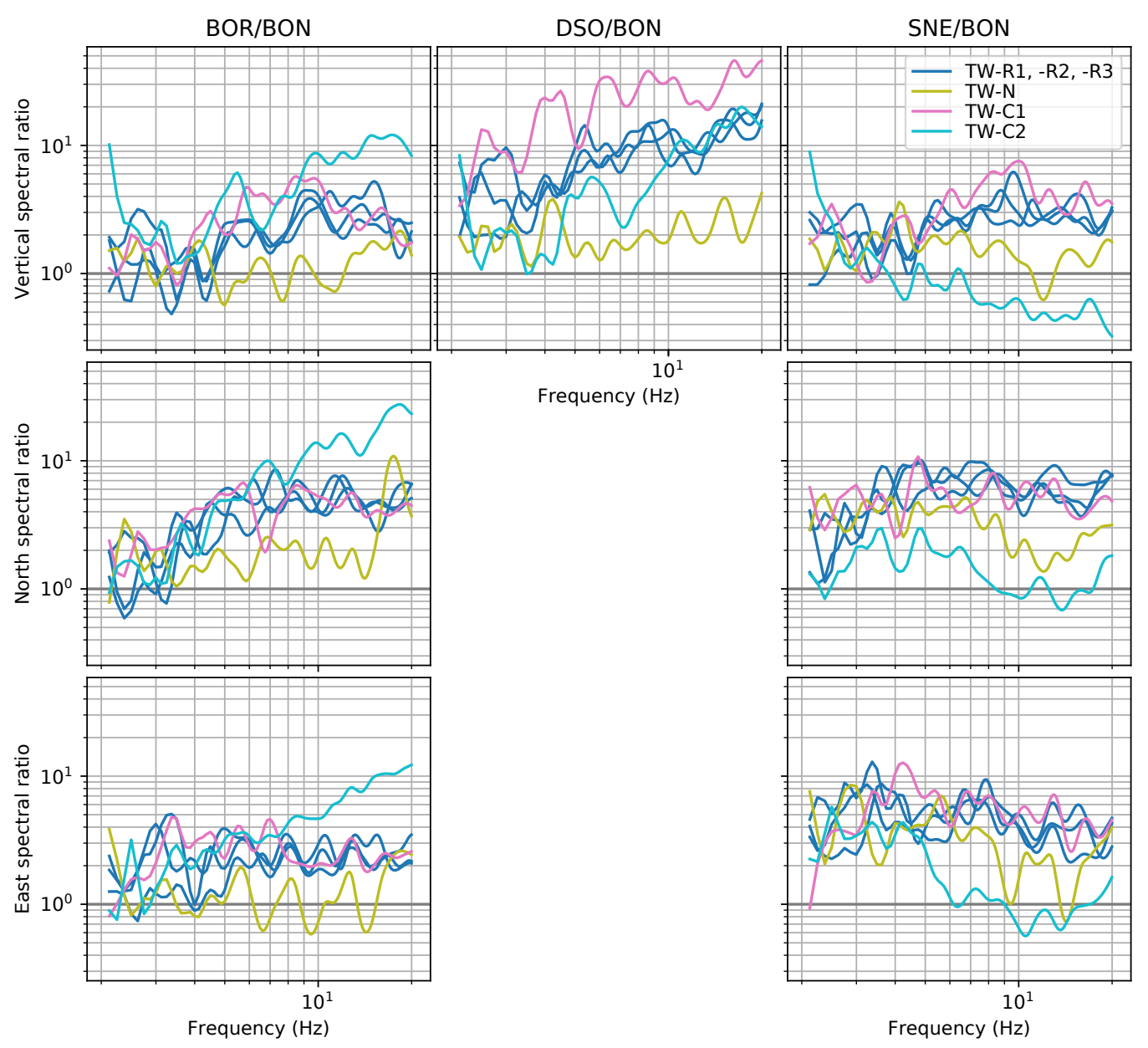

Figure 10. Spectral ratios from rockfall seismic signals recorded at stations BOR (3 components), DSO (1 component) and SNE (3 components) relative to station BON for vertical- (top), north- (middle) and east- (bottom) components. Time windows TW-R1, -R2, and -R3 correspond to rockfalls 1, 2, and 3 as defined in Figure 9. Time windows TW-N and TW-C1 correspond to noise recordings and the beginning of rockfall 1, respectively. Time window TW-C2 is taken from a rockfall on the southwestern crater wall, corresponding to rockfall location $\mathbf{2}$ in Figure 1 b.

curves $\mathrm{R} 1, \mathrm{R} 2$ and $\mathrm{R} 3$. This provides evidence that the spectral ratios are indeed characteristic of the position of the rockfall seismic source. The same analysis is carried out in Appendix D for rockfalls in the southwest, leading to the same conclusion.

\subsection{Comparison of observed and simulated spectral ratios}

The seismic source of a rockfall can be very complex as multiple impacts of different magnitude can occur simultaneously at different positions. Hence, it is very difficult to correctly simulate the rockfall seismic signal, especially at high frequencies. For this reason, spectral ratios between stations are very convenient to compare real and synthetic signals. In this way, the signature of the source is removed from the signal and solely propagation path effects remain. Nevertheless, we have to keep in mind two points when comparing observed and simulated spectral ratios.

Firstly, local subsurface heterogeneities can modify recorded amplitudes and thus influence inter-station ratios. These geological site effects are not considered in the sim- 
ulations. Therefore, in order to enhance comparability between the observed and simulated spectral ratios, the recorded signals are corrected using site amplification factors estimated from volcano-tectonic (VT) signals. The spectral amplification curves are calculated and discussed in Appendix $\mathrm{C}$ where we also show a comparison between simulated and uncorrected observed spectral ratios. The observed inter-station ratios presented here are corrected by deconvolution of the recorded signals with the corresponding amplification factors.

Secondly, different source directions cause different radiation patterns. This is illustrated in Figure 11a, where a force on a flat surface is polarized in vertical (top) and horizontal (bottom) directions. If the radiation pattern is not radially symmetric, which is only the case for vertical ground motion from a vertical source, the spectral ratios are affected depending on the azimuthal position of the respective receivers. The direction of a rockfall seismic source depends both on the rockfall dynamics and on the underlying slope. The generated forces from a boulder impact are schematically illustrated in Figure $11 \mathrm{~b}$. The resulting force $F_{r}$ is composed of a force $F_{n}$ normal to the slope and a force $F_{t}$ tangential to the slope, which depends on the slope angle, the direction of movement and the friction between the moving mass and the ground.

In order to analyze the influence of the source direction on the spectral ratios, we compare a vertical force to a normal force and a tangential force. Note that we assume that the tangential force is parallel to the slope of steepest descent. To consider a spatially distributed source in the simulations, the mean spectral ratio is calculated from a selection of multiple sources. This makes it possible to simultaneously evaluate the sensitivity of the curves to the source positions. Seven source positions are picked from a grid with $10 \mathrm{~m}$ spacing (see Fig. 1b, picked sources). The area corresponds to the region in which rockfalls 1,2 , and 3 are present during time windows R1, R2, and R3, respectively (see Fig. 9).

\subsubsection{Simulated spectral ratios for a model with a flat surface}

Figure 11c compares spectral ratios BOR/BON from the observed signals with simulated ratios of differently polarized sources on a model with a flat surface. The source directions are determined from the slope of Dolomieu topography at the corresponding position before implementation on the flat domain. For spectral ratios of the vertical component (left), a tangential force direction results in much smaller values compared to the other sources. As the slope dips northwards, the tangential force is orientated in the northdirection. Station BOR is located west of the source position, which is transverse to the source direction. For this reason, a smaller signal amplitude is measured at station BOR in comparison with station BON (ratio $<1$ ), even though BOR is slightly closer to the source. Nevertheless, the tangential force also contains a vertical component that ensures that the ratio is of the same magnitude as the observed ratios. This is different for the spectral ratios of the north component (middle), where a striking discrepancy of more than one order of magnitude results from the vertical source. A vertical force does not generate horizontal transverse energy which is why almost no signal is recorded on the north component at station BOR located eastwards. For the east spectral ratios (right), the tangential force again shows the strongest deviation for reasons similar to those for the vertical component spectral ratios.

\subsubsection{Simulated spectral ratios for a model with topography}

As opposed to the model with a flat surface, a model with the Dolomieu crater topography results in a good agreement between simulated and observed spectral ratios (see Figure 12). Furthermore, very similar values can be observed when comparing the simulations with different source directions, especially towards higher frequencies. This indicates that the spectral ratios are in this case not dominated by the direction of the 
a)
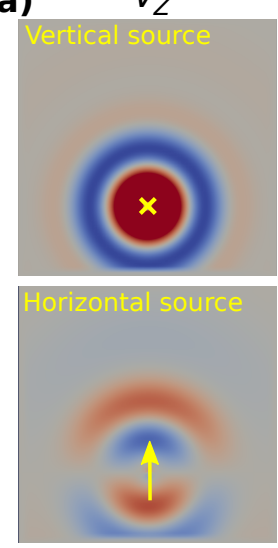
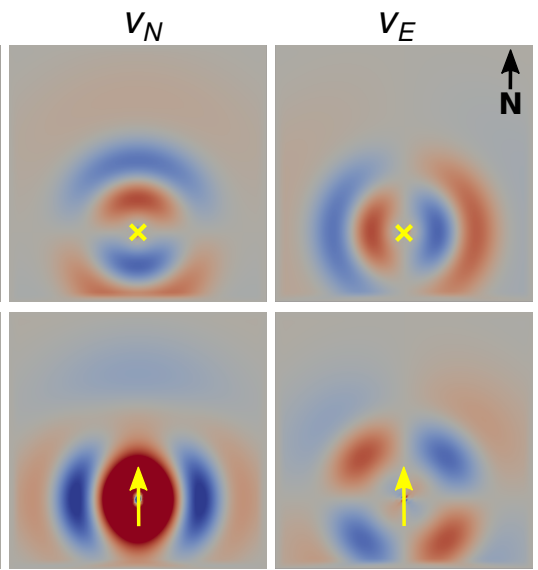

b)

c)
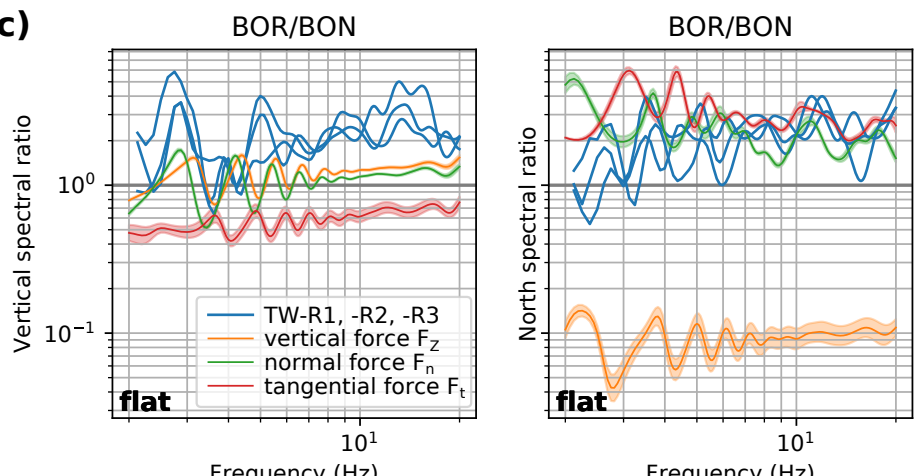

Frequency $(\mathrm{Hz})$
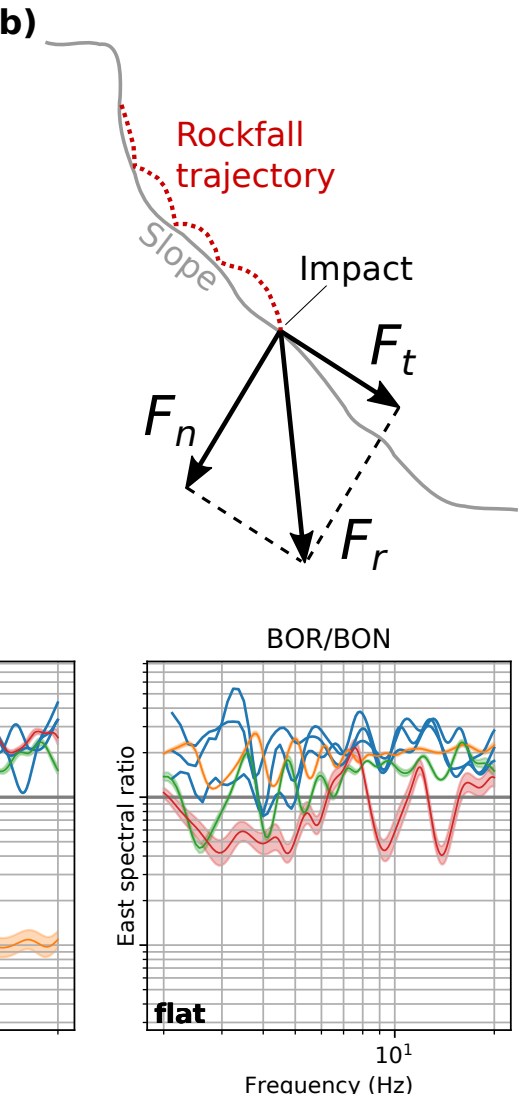

Frequency $(\mathrm{Hz})$

Figure 11. a) Seismic radiation patterns from a vertical source (top) and from a horizontal force (bottom) for ground velocity $v_{Z}, v_{N}$, and $v_{E}$ of vertical-, north-, and east-component, respectively (red for positive and blue for negative amplitudes). b) Forces generated by a rockfall impact. The red dotted line illustrates the trajectory of a bouncing boulder. The impact generates force $F_{n}$ normal to the slope. Depending on the boulder velocity tangential to the slope and on the friction coefficient $\mu$, a tangential force $F_{t}=\mu F_{n}$ is generated (assuming Coulomb friction). Normal and tangential forces add up to resulting force $F_{r}$. c) Comparison of spectral ratios BOR/BON from real signals TW-R1, -R2, -R3 (as in Fig. 10, site-effect corrected) and from simulations on the flat domain with varying source direction: vertical force, normal force and tangential force according to the Dolomieu topography at the corresponding position. The shaded zones of the simulated ratios indicate the standard deviation around the mean value from seven neighbouring source positions (Fig. 1b, picked sources). SEM configurations correspond to 15 in Table A1.

source (and the corresponding produced radiation pattern) but rather by propagation along the topography.

Greater deviations between the different source directions are found at lower frequencies, such as for example on the north component of ratio BOR/BON below $3 \mathrm{~Hz}$. Assuming fundamental Rayleigh waves, this corresponds to wavelengths above $250 \mathrm{~m}(\lambda \approx$ $750 \mathrm{~m} \mathrm{~s}^{-1} \div 3 \mathrm{~Hz}$ ). With a distance of around $500 \mathrm{~m}$ between the source position and station BOR, it is likely that these low-frequency waves have not traveled enough wavelengths in order to be completely dominated by propagation along the topography.

Analyzing the sensitivity of the ratios to the source position, generally larger standard deviations (shaded zone of uncertainty around the mean) are present after intro- 


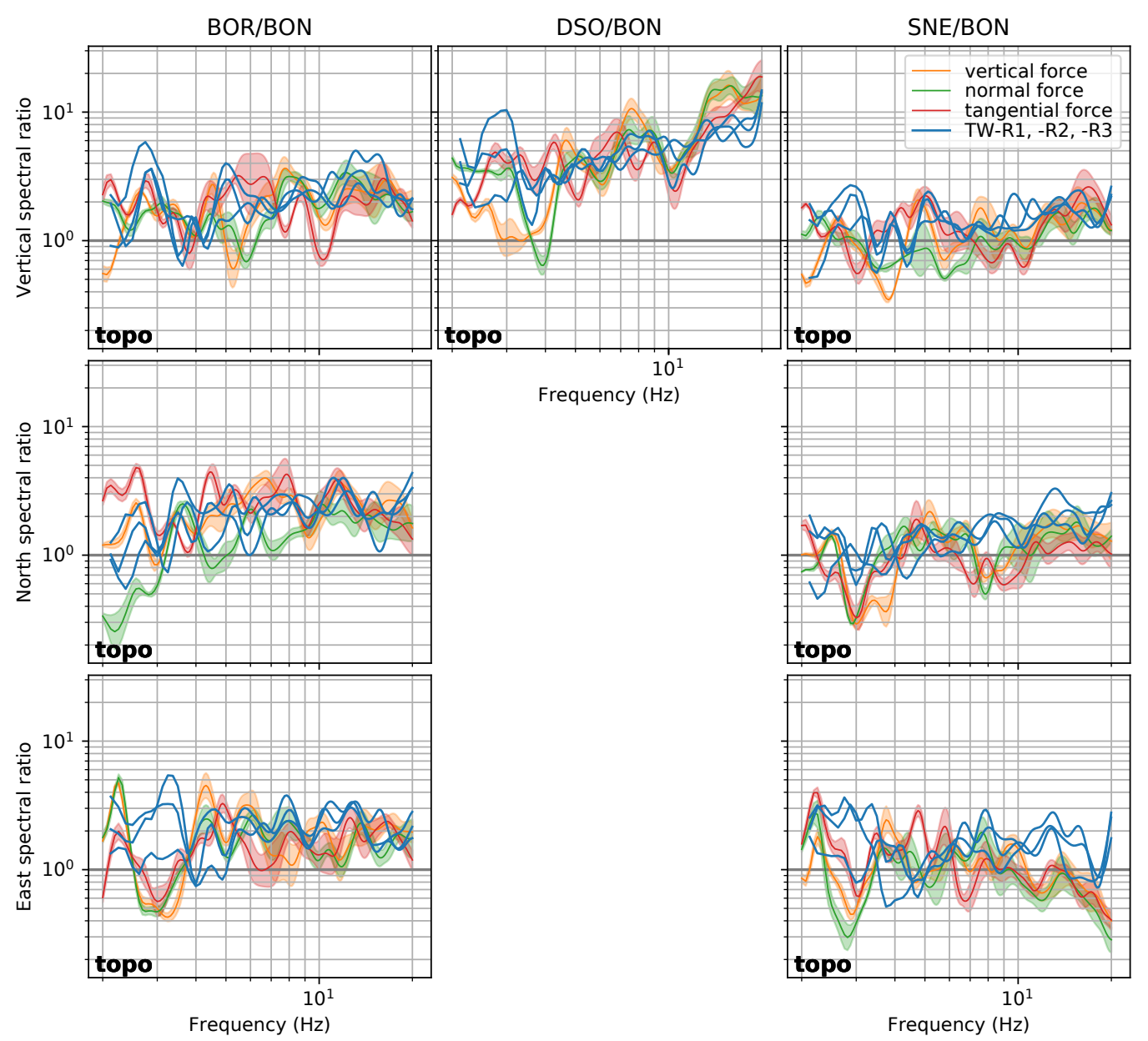

Figure 12. Spectral ratios BOR/BON, DSO/BON and SNE/BON calculated from real signals TW-R1, -R2, -R3 (as in Fig. 10, site-effect corrected) and from simulations on the domain with Dolomieu topography for the vertical (top), the north (middle), and the east (bottom) component. Simulations are carried out with varying source directions: vertical force, force normal to the slope and force tangential to the slope. The shaded zones of the simulated ratios indicate the standard deviation around the mean value from seven neighbouring source positions (Fig. 1b, picked sources). SEM configurations correspond to 16 in Table A1.

ducing topography compared to the results for the flat model in Figure 11c. This means that a slight change of source position allows more variability of the ratios when considering topography and can eventually better explain the observed spectral ratios.

Clearly, the spectral ratios also depend on the relative source-receiver distance. For example, the high values of ratio $\mathrm{DSO} / \mathrm{BON}$ result from the fact that the source is very close to station DSO. Furthermore, the values increase towards higher frequencies. This is related to the attenuating properties of the medium that cause the amplitudes of higher frequencies to decrease faster with the distance traveled than lower frequencies.

The analysis suggests that the spectral ratios are characteristic of the source position and dominated by propagation along the topography rather than by the radiation patterns caused by the source directions. To further validate this hypothesis, the same comparison between observations and simulations is carried out in Appendix D for rockfalls located on the southwestern crater wall. 
a)

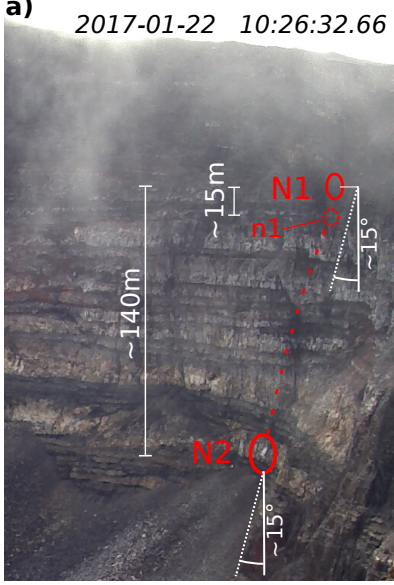

b)

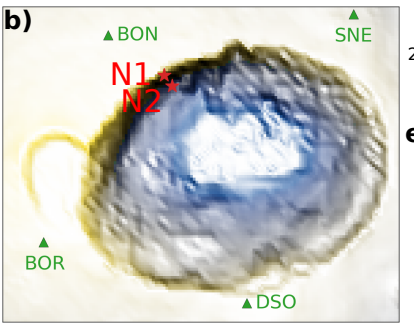
ity, and amplitudes.

\subsection{Seismic signature of a rockfall impact}

We will now analyze in detail the seismic signal generated by the single impacts of a rockfall at Dolomieu crater. Interpretation of the signal characteristics is based on comparison with synthetic signals simulated on models with and without topography. The comparison between observed and simulated signals has to be carried out very carefully because of the uncertainties of the seismic source and the propagation medium. It is important to emphasize that we do not want to reproduce the recorded signal but rather understand some of its features, such as for example arrival times, waveform complex-

For the analysis, a single boulder rockfall is chosen with well separated impacts that can be tracked on video. These criteria are fulfilled by an event that occurred on January 22, 2017, located on the northern crater wall. Figure 13 shows a camera snapshot of the rockfall at the time of impact N2 as well as the impact locations and the rockfall seismic signal recorded for the vertical component at the closest station BON. Two boul-

c)
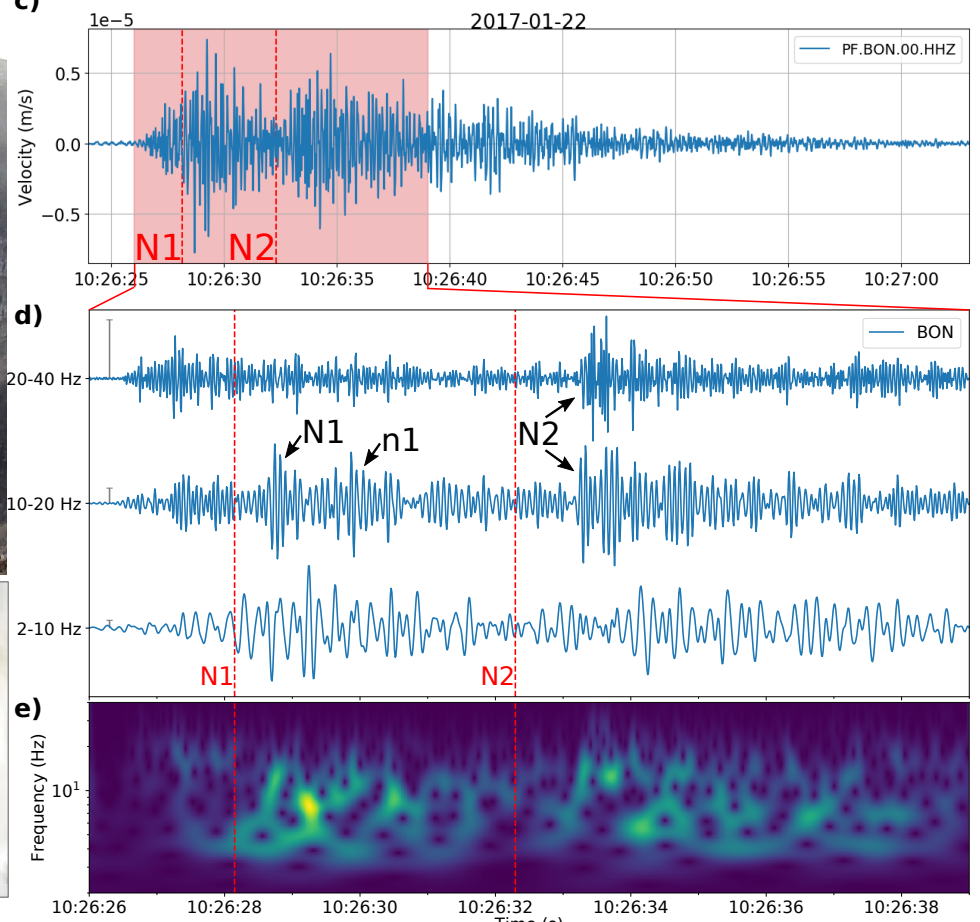

Figure 13. Single boulder rockfall on January 22, 2017. a) Camera snapshot taken shortly after impact N2. Estimated vertical distance between impacts and estimated slope angle to the vertical at impact positions. b) Location of impacts N1 and N2 in Dolomieu crater. c) Vertical ground velocity recorded at closest station BON in frequency band 2-40 Hz. The red-shaded area illustrates the time window of the graph below. Dashed lines mark impact times N1 and N2 estimated from the video. d) Comparison of frequency bands $2-10 \mathrm{~Hz}, 10-20 \mathrm{~Hz}$, and $20-40 \mathrm{~Hz}$. Signals are normalized to their maximum and the gray bars on the left indicate the relative scaling. e) Time-frequency representation of the rockfall signal (calculated using the Stockwell transform).

der impacts, $\mathrm{N} 1$ and N2, around $4 \mathrm{~s}$ apart, are analyzed. A minor impact $\mathrm{n} 1$ is observed $1 \mathrm{~s}$ after impact N1. It will be used later to estimate the fall velocity of the boulder. Note that the impact times are estimated from the video according to the appearance of small 
dust clouds caused by the impacts. The time delay between the actual impact and the visibility of the dust cloud influences the accuracy of the impact time to a similar order of magnitude as the sampling time of $0.5 \mathrm{~s}$ between successive snapshots.

The broadband seismic signal of the rockfall shown in Figure 13c is characterized by two main lobes. These lobes are separated by a gap of low seismic energy at around 10:26:32. During this gap, no impact is observed on the video. Thus, the boulder is in free fall before hitting the ground at impact location N2. Afterwards, the rockfall splits into several blocks that continue to move downwards on the debris cone of former rockfalls. At these later times it is very difficult to identify single impacts.

To better distinguish single impacts, the seismic signal is filtered in different frequency bands. Figure 13d compares signals band-pass filtered at $2-10 \mathrm{~Hz}, 10-20 \mathrm{~Hz}$, and $20-40 \mathrm{~Hz}$. The relative scales of the normalized signals can be inferred from the gray bars plotted at the beginning of the signal as well as from the spectrogram below.

The signal filtered in the low frequency band $(2-10 \mathrm{~Hz})$ exhibits a smooth amplitude envelope. The two main lobes discussed above can be observed whereas no single pulses can be identified. This signal contains the strongest amplitudes and thus dominates the broadband signal. Short signal pulses emerge in the high-frequency bands. It is clear that seismic sources were already active before impact N1. Unfortunately, they could not be detected on the video. Impacts are possibly hidden behind the clouds on the top of the crater wall. A clear seismic pulse in the frequency range $10-20 \mathrm{~Hz}$ can be ascribed to impact $\mathrm{N} 1$. It arrives at the station around $0.5 \mathrm{~s}$ after the time determined from the video. A second pulse around $1 \mathrm{~s}$ later can be ascribed to impact n1. It contains slightly smaller amplitudes. The highest frequency band does not show signals clearly corresponding to these two impacts. This is different for impact N2. Both high-frequency bands show abrupt signal onsets around 1s after the detection of impact N2 on the video. The following signal cannot be described as a single pulse but contains several peaks. This raises the question as to whether the source is made up of several impacts or if these peaks result from seismic wave propagation.

Another interesting observation concerns the impact-generated frequencies. As we can see, impact N1 is barely detectable in the highest frequency range $(20-40 \mathrm{~Hz})$, whereas impact N2 produces clear signals in both high-frequency bands $(10-20 \mathrm{~Hz}$ and $20-40 \mathrm{~Hz})$. Considering the changing source-receiver distance, we would expect the contrary as N1 is slightly closer to station BON than N2. If we assume that the properties of the boulder and of the underlying ground are identical for both impacts, the change in frequency content must be related to the impact velocity. As the boulder accelerates between impact N1 and impact N2, the higher velocity at impact N2 results in a shorter collision time and therefore generates higher frequencies according to Hertz contact theory, introduced below.

\subsubsection{Hertz contact theory}

To predict relative amplitudes of signals generated by impacts N1 and N2, the respective impact forces of the boulder on the ground are estimated. Farin et al. (2015) used the theory of Hertz (1878) to describe the force of an elastic sphere impacting a solid elastic surface. After successfully applying the theory on seismic signals generated in laboratory experiments, they analyzed real-size rockfall experiments carried out by Dewez et al. (2010). Here we estimate the impact forces in a similar fashion, assuming a spherical boulder of radius $R$ and mass $m$, where $m=\rho 4 / 3 \pi R^{3}$ with rock density $\rho$. The maximum impact force $F_{0}$ exerted by the sphere perpendicularly to the plane can then be expressed as (Johnson, 1989)

$$
F_{0}=\frac{4}{3} E R^{1 / 2} \delta_{\max }^{3 / 2}
$$

where $\delta \max$ is the maximum indentation depth

$$
\delta_{\max }=\left(\frac{15 m v_{n}^{2}}{16 E R^{1 / 2}}\right)^{2 / 5},
$$


with impact speed $v_{n}$ normal to the plane. $E$ is the effective Young's modulus $1 / E^{*}=$ $\left(1-\nu_{s}^{2}\right) / E_{s}+\left(1-\nu_{p}^{2}\right) / E_{p}$, where $\nu_{s}, \nu_{p}, E_{s}$, and $E_{p}$ are Poisson's ratio and Young's modulus of a sphere and an impacted plane, respectively.

Concerning the frequency content of the impacts, we analyze the contact duration of the impacts. As proposed by Johnson (1989), the temporal evolution of the Hertzian impact force $F_{H}$ can be approximated by

$$
F_{H}(t) \approx F_{0} \sin \left(\pi t / T_{c}\right)^{3 / 2}, \quad 0 \leq t \leq T_{c} .
$$

The force-time function and its frequency spectrum are shown in Figure 14 as a function of impact duration $T_{c}$.
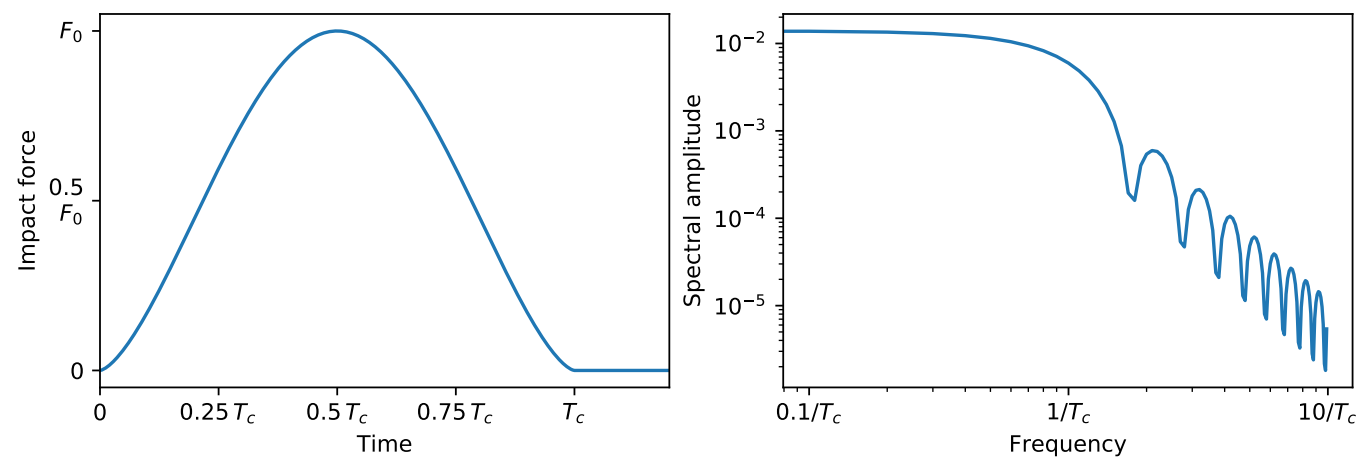

Figure 14. Hertzian impact force and corresponding frequency spectrum. Left: Hertzian force-time function $F_{H}$ normalized by maximum impact force $F_{0}$ versus impact duration $T_{c}$, which represents the time during which the two bodies are in contact. Right: Frequency spectrum of the force-time function. The inverse impact time $1 / T_{c}$ is related to the corner frequency $f_{c}$ after which the spectral amplitude decays in the form of a power law.

The spectral amplitude decays in the form of a power law above corner frequency $f_{c}=1 / T_{c}$. Johnson (1989) showed that the impact duration can be approximated by means of maximum indentation depth $\delta_{\max }$ and impact normal speed $v_{n}$ as

$$
T_{c} \approx 2.94 \frac{\delta_{\max }}{v_{n}} .
$$

The values of impact parameters for N1 and N2 are estimated assuming that boulder and ground properties are identical between the two impacts. After careful consideration, the uncertainties for all parameters is uniformly set to $\pm 50 \%$, which is found to be reasonable as a maximum threshold from a physical perspective and also allows numerical comparison of the respective contribution of each parameter on the total uncertainty of impact force and corner frequency.

To estimate impact speed $v_{n}$ normal to the slope, a sub-vertical fall of the boulder before collision is assumed for both $\mathrm{N} 1$ and $\mathrm{N} 2$ with vertical speed $v_{c}$ at the time of collision. Slope angles at the impact positions are inferred from the DEM to be around $\alpha=15^{\circ}$. The normal impact speed can then be calculated as $v_{n}=v_{c} \sin \alpha$. To estimate $v_{c}$ for $\mathrm{N} 1$ and $\mathrm{N} 2$, height differences between the impacts are determined from the DEM using the impact positions estimated from the video. As labeled in Figure 13a, we find a height difference of around $H_{1}=15 \mathrm{~m}$ between $\mathrm{N} 1$ and $\mathrm{n} 1$ and a height difference of around $H_{2}=140 \mathrm{~m}$ between $\mathrm{N} 1$ and $\mathrm{N} 2$. Impacts $\mathrm{N} 1$ and $\mathrm{n} 1$ are detected $1 \mathrm{~s}$ apart. Assuming an approximately constant velocity during this short time window, the vertical speed for impact $\mathrm{N} 1$ is $v_{c, 1}=15 \mathrm{~m} \mathrm{~s}^{-1}$. For impact N2, acceleration during the long free fall cannot be neglected. The speed is thus derived by $v_{c, 2}=v_{c, 1}+\left(2 g\left(H_{2}-\right.\right.$ 
a)

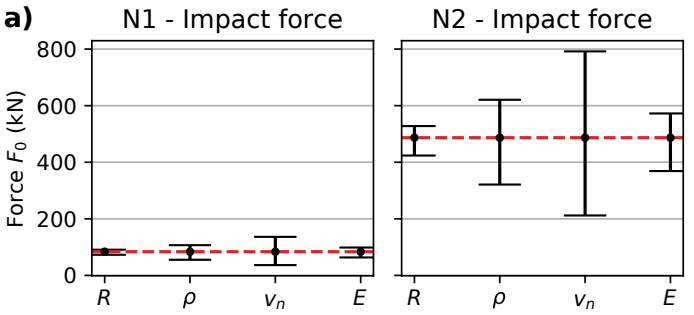
and $v_{n, 1}=17 \mathrm{~m} \mathrm{~s}^{-1}$ for impact $\mathrm{N} 1$ and $\mathrm{N} 2$, respectively. $10 \mathrm{MPa}$ is applied as proposed by Farin et al. (2015).

$\left.\left.H_{1}\right)\right)^{0.5}$, where $g=10 \mathrm{~m} \mathrm{~s}^{-2}$ is acceleration due to gravity. Hence, a vertical speed $v_{c, 2}=$ $65 \mathrm{~m} \mathrm{~s}^{-1}$ is found for impact N2. This leads to normal impact speeds of $v_{n, 1}=4 \mathrm{~m} \mathrm{~s}^{-1}$

The boulder size is approximated from camera snapshots. The dust cloud caused by impact N2 in Figure 13a has an estimated length of $5 \mathrm{~m}$. As only the dust clouds and not the boulder itself can be seen on the video, the boulder size is assumed to be less than $1 \mathrm{~m}$. As a lower bound, a size bigger than $0.2 \mathrm{~m}$ is assumed necessary to generate a seismic signal clearly above the ambient noise level. We therefore estimate the boulder size to be the mid-point between these two limits, i.e. $0.6 \mathrm{~m}$. As a perfect sphere is considered in the calculations, the effective radius is hence estimated to be $R=0.3 \mathrm{~m}$.

The rock density is estimated to be $\rho=2000 \mathrm{~kg} \mathrm{~m}^{-3}$, as in the simulations, which results in a boulder mass of $m=226 \mathrm{~kg}$. A typical effective Young's modulus of $E=$

The maximum impact force $F_{0}$ can now be calculated using equation 3 . We find $84 \mathrm{kN}$ and $487 \mathrm{kN}$ for impacts $\mathrm{N} 1$ and $\mathrm{N} 2$, respectively. The maximum deviations, summarized in Table 2 and ranging from $-95 \%$ to $+450 \%$, are estimated numerically by varying each parameter by $\pm 50 \%$. Figure 15 a breaks down the contribution of each parameter to the maximum uncertainty. Note that the relative errors are the same for both impacts $\mathrm{N} 1$ and $\mathrm{N} 2$. We can observe that a variation of impact speed $v_{n}$ has the greatest effect on the impact force, followed by the rock density $\rho$.

Table 2. Hertz impact parameters ${ }^{b}$

\begin{tabular}{cccccccc}
\hline & $v_{c}$ & $\alpha$ & $v_{n}$ & $\delta_{\max }$ & $F_{0}$ & $T_{c}$ & $f_{c}$ \\
\hline $\mathrm{N} 1$ & $15 \mathrm{~m} \mathrm{~s}^{-1}$ & $15^{\circ}$ & $4 \mathrm{~m} \mathrm{~s}^{-1}$ & $0.05 \mathrm{~m}$ & $\left(84_{-79}^{+376}\right) \mathrm{kN}$ & $0.038 \mathrm{~s}$ & $\left(26_{-16}^{+61}\right) \mathrm{Hz}$ \\
$\mathrm{N} 2$ & $65 \mathrm{~m} \mathrm{~s}^{-1}$ & $15^{\circ}$ & $17 \mathrm{~m} \mathrm{~s}^{-1}$ & $0.16 \mathrm{~m}$ & $\left(487_{-460}^{+2186}\right) \mathrm{kN}$ & $0.029 \mathrm{~s}$ & $\left(35_{-21}^{+82}\right) \mathrm{Hz}$ \\
\hline
\end{tabular}

${ }^{b}$ Parameters for impacts $\mathrm{N} 1$ and N2: vertical impact speed $v_{c}$, angle $\alpha$ between the slope and the vertical, impact speed $v_{n}$ normal to the slope, maximum indentation depth $\delta_{\max }$, impact force $F_{0}$ (with maximum deviations), contact time $T_{c}$, and corner frequency $f_{c}$ (with maximum deviations).

Figure 15. Influence of individual parameters on uncertainty of impact force $F_{0}(\mathbf{a})$ and corner frequency $f_{c}(\mathbf{b})$. Impact parameters include boulder radius $R$, rock density $\rho$, normal impact speed $v_{n}$ and Young's modulus $E$. Uncertainties are estimated numerically by varying each parameter by $\pm 50 \%$. Note that the relative errors for $\mathrm{N} 1$ and $\mathrm{N} 2$ are identical.

The impact duration is estimated to be $0.038 \mathrm{~s}$ and $0.029 \mathrm{~s}$ for $\mathrm{N} 1$ and $\mathrm{N} 2$, respectively. As $f_{c}=1 / T_{c}$, it follows that the high-frequency content of the impacts are lim- 
ited by corner frequency $26 \mathrm{~Hz}$ and $35 \mathrm{~Hz}$, respectively, with maximum deviations ranging from $-62 \%$ to $+236 \%$, see Table 2 . The contribution from each parameter is indicated in Figure 15b for impacts N1 and N2. In contrast to the impact force, the frequency content is least sensitive to the normal impact speed $v_{n}$. It is most sensitive to rock density $\rho$ and Young's modulus $E$.

An important result is that Hertz contact theory predicts a higher frequency content for N2, related to the higher impact velocity. This agrees with the observed waveforms in Figure 13d: impact N1 can hardly be detected in the high-frequency band (20$40 \mathrm{~Hz}$ ), whereas impact N2 shows a clear pulse despite the slightly bigger source-receiver distance. The theoretical values agree well with the observations, predicting frequencies up to $26 \mathrm{~Hz}$ and $35 \mathrm{~Hz}$ for $\mathrm{N} 1$ and $\mathrm{N} 2$, respectively.

\subsubsection{Comparison of observed and synthetic waveforms}

Previously, we tried to associate pulses in the observed seismic signal to impacts observed in the video by crudely interpreting the signal after the time of impact. We will now use numerical simulations to obtain insights into travel times and expected waveforms. As already mentioned, the intention is not to reproduce observed waveforms, but rather to understand which signal characteristics can be ascribed to a single impact.

Observed and synthetic signals are compared in the frequency band of $10-20 \mathrm{~Hz}$, in which we identify short signal pulses caused by the rockfall impacts. At the same time, $20 \mathrm{~Hz}$ constitutes the upper frequency limit of our simulations. In order to ensure comparability, the observed signals are corrected with the site amplification functions calculated in Appendix $\mathrm{C}$ and subsequently convolved with the $7 \mathrm{~Hz}$ Ricker wavelet used in the simulations. The simulated signals are calibrated with the maximum impact forces for N1 and N2 estimated above using Hertz theory.

Initially, observed and synthetic seismograms are normalized for easier comparison of the waveforms. Figure 16 compares vertical ground velocity recorded at stations BON, BOR, DSO, and SNE with simulations from impacts N1 and N2 for models with a flat surface and with Dolomieu topography. Source positions of the two impacts are estimated from the videos, see Figure 13b. As the exact source direction of the real impacts are unknown, the variability of synthetic waveforms is shown for different force directions, namely a vertical force $F_{z}$, a force $F_{n}$ normal to the slope and a force $F_{t}$ tangential to the slope.

Analyzing the synthetic seismograms, it can generally be observed that N1 produces smaller amplitudes compared to N2. This is due to the estimated impact forces of $84 \mathrm{kN}$ and $485 \mathrm{kN}$, respectively (see Table 2).

While seismograms from the model with a flat surface keep approximately the same relative amplitudes between $\mathrm{N} 1$ and $\mathrm{N} 2$ at all different stations, seismograms from the model with topography show more variability. For example, at station BON, the amplitudes of impact N1 are much bigger for the model with topography than for the flat model. This corresponds better to the real observations, where the maximum amplitude of impact N1 is comparable to the maximum amplitude of impact N2. In contrast, at station DSO, the signal of impact N1 is very small as opposed to the signal of N2 for the model with topography. Again, this corresponds well with the observations. As impacts $\mathrm{N} 1$ and N2 are located very close to each other, the relative amplitudes on the flat model are mainly determined by the relative impact force. In contrast, surface topography influences both the relative (vertical) source position and the propagation path, so that small source displacements can cause local amplification or deamplification of the signal. Measuring the signal at station DSO, the source moves from a deamplified zone at $\mathrm{N} 1$ towards an amplified zone at N2, as suggested by the reciprocal amplification pattern provided in the Supporting Information.

From the simulations with a flat surface, three wave packets can be observed following each impact, which are well separated from each other on the more distant stations BOR, DSO, and SNE. These three wave packets correspond to a body wave, a first- 

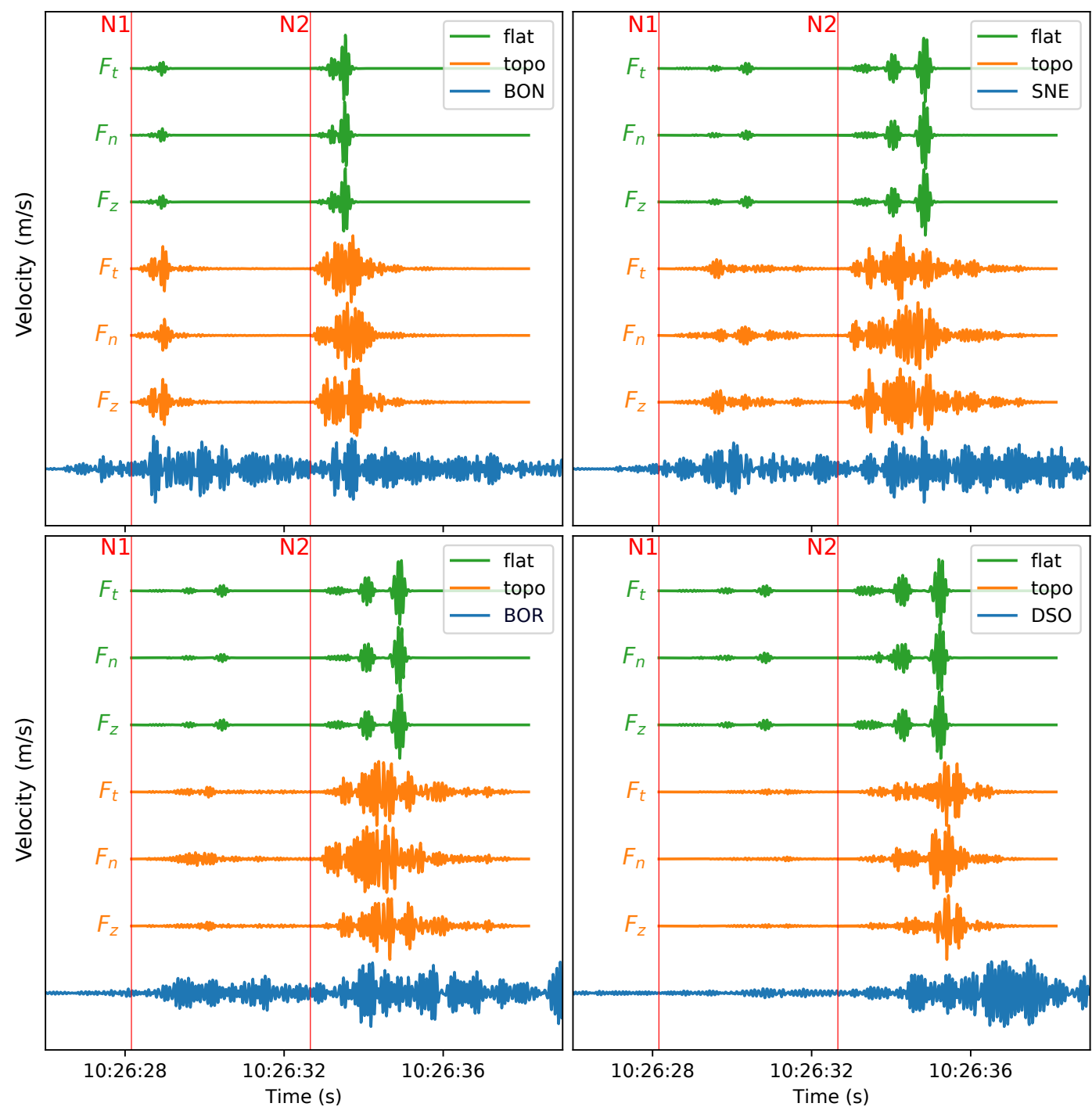

Figure 16. Comparison of recorded rockfall signal (blue, for a time window as in Figure 13d) with synthetic waveforms of impacts N1 and N2 for the model with a flat surface (green) and with Dolomieu topography (orange). The variability of the synthetic waveforms is demonstrated for a vertical force $F_{z}$, a force $F_{n}$ normal to the slope and a force $F_{t}$ tangential to the slope. Red vertical lines indicate impact times N1 and N2 from the video. Corresponding source positions are shown in Figure 13b. All signals show vertical ground velocity and are normalized by their maximum. SEM configurations correspond to 15 and 16 in Table A1.

mode Rayleigh wave, and a fundamental mode Rayleigh wave (see e.g. Figure 3). The arrival time of the first-mode Rayleigh wave is in good agreement with the first major pulse after each impact. This suggests that the Lesage generic velocity model represents the shallow subsurface velocity around Dolomieu crater reasonably well. However, for the flat model, the amplitude of the first-mode Rayleigh wave is consistently smaller than the amplitude of the fundamental mode. A corresponding amplitude variation cannot be identified on the real signals. In contrast, simulations on the model with topography generate more complex waveforms. This increased complexity corresponds better to the observed signals, even if the waveforms do not fit perfectly. The variation of the force direction modifies the waveforms more than in the flat case. Also, waveforms vary greatly 
from station to station. This is not observed in the flat case, in which the waveforms are very similar for stations at comparable source-receiver distances (i.e. BOR, DSO, and SNE). Similar observations are made for synthetic signals generated by neighboring source positions (10 m spacing), exhibiting much more variability for the case of topography, see Supporting Information.

It cannot be excluded that the observed amplitude variations and waveform complexity can be explained by heterogeneous velocity structures or the superposition of multiple (here undetected) sources. Nonetheless, the simulations indisputably show that these effects are caused by surface topography, for which high-resolution models are available. Consequently, surface topography must be taken into account when interpreting highfrequency rockfall seismic signals and multiple signal pulses must not directly be interpreted to be caused by multiple impacts.

Finally, observed and synthetic seismograms are compared without normalization. In this way, the absolute signal amplitudes calibrated by the Hertz impact force are evaluated. The total value of the acting force as well as its direction are determined by a vector sum of the forces normal and tangential to the slope. Tangential force $F_{t}$ is inferred from the maximum normal impact force $F_{n}=F_{0}$ assuming Coulomb friction $F_{t}=\mu F_{0}$, where $\mu$ is the material-specific friction coefficient. We assign $\mu=0.7$, a typical value used for rockfalls at Dolomieu crater (e.g. Hibert, Mangeney, et al., 2014). The resulting signal amplitudes for model simulations with a flat surface and with topography are compared with the observed rockfall signals in Figure 17.
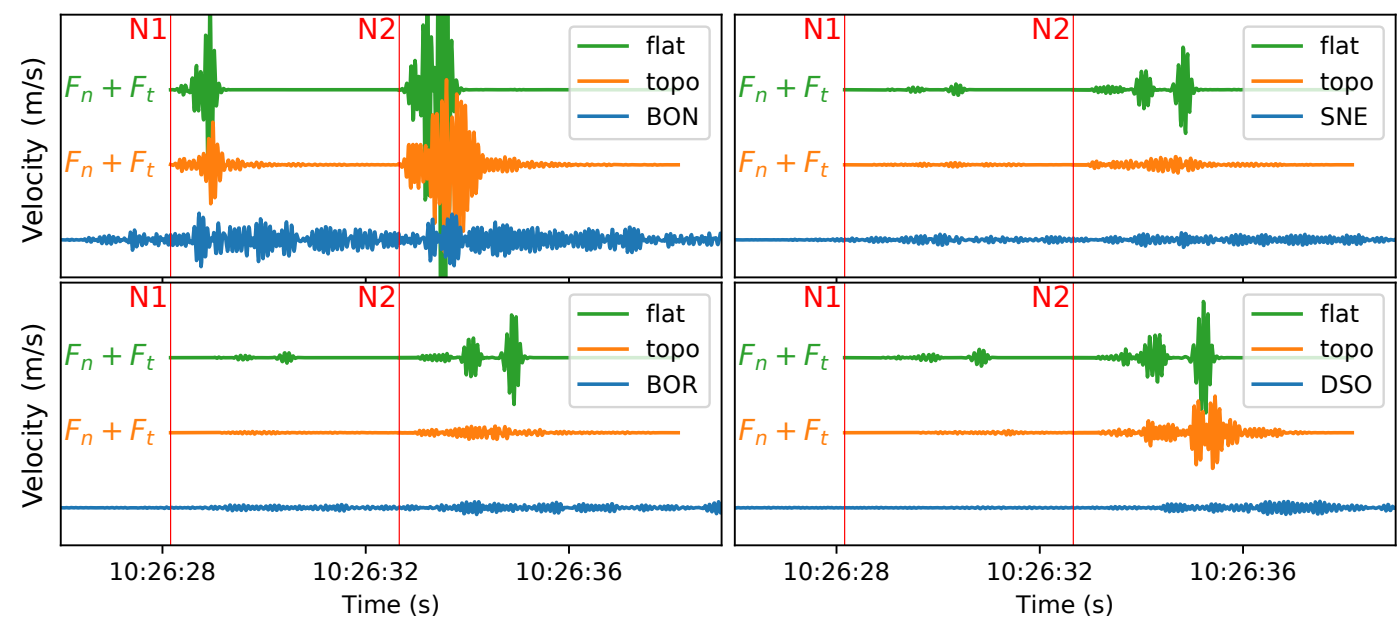

Figure 17. Amplitude comparison of recorded rockfall signal (blue, for a time window as in Figure 13d) with synthetic waveforms of impacts N1 and N2 for the model with a flat surface (green) and with Dolomieu topography (orange). The synthetic seismic source is constructed by summing force $F_{n}$ and $F_{t}$ normal and tangential to the slope. $F_{n}$ corresponds to the maximum impact force $F_{0}$ as shown in Table 2. Red vertical lines indicate impact times N1 and N2 from the video. Corresponding source positions are shown in Figure 13b. All signals show vertical ground velocity and are normalized by their maximum. SEM configurations correspond to 15 and 16 in Table A1.

Despite the uncertainties on impact force $F_{0}$ and the assumptions on elastic contact and Coulomb friction, which are certainly violated by a certain amount of plasticity and surface roughness, we are able to achieve order-of-magnitude agreements with the observed signals. The application of the Hertz contact theory to calibrate the simulations therefore offers a solution to this poorly constrained problem. 
When comparing the amplitudes between the model with a flat surface and the model with topography, one notices that the relative amplitude changes between the stations. While amplitude decreases linearly with the distance from the source for the flat model, this is not the case for simulations with topography. For example, for the flat model, the maximum amplitude decreases drastically from station BON to station DSO. In contrast, for the model with topography, the signal amplitude of impact N2 at station DSO is only about half that of station BON, which can be assigned to topographic amplification.

\section{Conclusion}

Using Spectral-Element-based simulations with high-resolution surface topography, we were able to broadly explore the influence of topography on the propagation of wave fields generated by surface point loads and to achieve order-of-magnitude agreement with seismic measurements from rockfalls at Dolomieu crater.

In the first half of the present study we numerically simulated the topography effect for different earth models. It was shown that the topography effect is significantly altered depending on the underlying velocity model. For example, for a homogeneous model, seismic energy is easily directed downwards by the topography into the subsurface. This is contrary to a velocity-depth profile with a strong gradient, as proposed by Lesage et al. (2018) for the shallow velocity structure of volcanoes, for which more seismic energy is trapped close to the surface leading to more complex spatial distributions of amplification.

Studying the geometric features of Dolomieu-like crater topographies suggests that curvature variations affect the seismic wave field more than depth variations. However, the locally inherent complexity of the topography prohibits a holistic generalization of its effect on the seismic wave field. Nevertheless, our approach provides a methodology to quantify the influences of the topography. This methodology can be used to other study sites by changing the model parameters regarding the domain surface and the medium properties, which are inevitably coupled. In this respect, scattering from 3D soil heterogeneities, which can strongly disturb wave propagation, must be considered. Regarding the study site at Dolomieu crater, previous studies and our findings indicate that scattering does not dominate the signals. Local site amplification at the stations could be taken into account by using amplification factors estimated from VT events, which significantly improved the agreement between simulations and observations. In general, however, it cannot be guaranteed that surface waves will receive the same amplification as a vertically incident wave field and it is recommended that wave propagation, including subsurface heterogeneities, be modeled when the necessary information is available.

In the second half of the present study, the simulations were compared with measurements from rockfalls at Dolomieu crater. Different rockfalls with similar impact locations were investigated by calculating spectral ratios between the stations, thereby extracting the signature of the seismic source. The agreement between the observed spectral ratios indicates identical path effects that were reproduced by simulations using the model with topography. It was further shown that the spectral ratios are dominated by propagation along the topography rather than by the direction of the seismic source. As the latter is hard to estimate precisely, this finding can have practical applications, for example for the locating of rockfalls.

Single impacts were shown to be able to generate complex waveforms with multiple pulses depending on medium properties and topography. For topography, variations of source direction and position can strongly modulate the waveforms.

Using Hertz contact theory, signal features were linked to impact parameters. Estimating the maximum impact force helped to calibrate the simulation and achieve orderof-magnitude agreement with observed signal amplitudes. Associating higher frequencies with increased impact speed explained the observed frequency content of rockfall impacts. 
The combination of Hertz contact theory and wave propagation simulations is an important step for the interpretation of rockfall seismic signals based on the underlying physical processes. The Hertz impact theory is frequently used to predict the impact force of rockfalls, for example for the design of protective structures (e.g. Volkwein et al., 2011). Also, laboratory experiments show the validity of Hertz theory concerning the waves generated by the collision of a ball on a massive plate (e.g. McLaskey \& Glaser, 2010) or grains on a plate (Bachelet et al., 2018). However, only a few studies apply the theory to seismic signals from real-scale rockfalls (Farin et al., 2015; Hibert, Malet, et al., 2017). A limiting factor is the complexity of the rockfall source that often consists of multiple simultaneous impacts. For this reason, application to artificially triggered rockfalls, ensuring separate impacts of a single boulder, would help validate the Hertz theory in the field and enhance our understanding of real impact processes.

\section{Appendix A Configuration of SEM simulations}

Table A1 contains all configurations of the SEM simulations conducted for the present paper and indicates the section in which each is used.

Table A1. Configuration of SEM simulations and usage in present $\operatorname{article~}^{c}$

\begin{tabular}{lllllll}
\hline & Velocity model & Surface & $\alpha$ & Source & Output & Sections \\
\hline 1 & homogeneous & Flat & Yes & $\mathrm{R} 1 ; Z$ & Seismograms & 4.1 \\
2 & homogeneous & Topo $20 \mathrm{~m}$ & Yes & $\mathrm{R} 1 ; Z$ & Seismograms & 4.1 \\
3 & homogeneous & Topo $20 \mathrm{~m}$ & $\mathrm{No}$ & $\mathrm{R} 1 ; Z$ & Snapshots & 3.4 \\
\hline 4 & low- $v_{S}$ layer & Flat & Yes & $\mathrm{R} 1 ; Z$ & Seismograms & 4.1 \\
5 & low- $v_{S}$ layer & Topo $20 \mathrm{~m}$ & Yes & $\mathrm{R} 1 ; Z$ & Seismograms & 4.1 \\
6 & low- $v_{S}$ layer & Topo $20 \mathrm{~m}$ & $\mathrm{No}$ & $\mathrm{R} 1 ; Z$ & Snapshots & 3.4 \\
\hline 7 & generic & Flat & Yes & $\mathrm{R} 1 ; Z$ & Seismograms & $3.3,4.1$ \\
8 & generic & Topo $20 \mathrm{~m}$ & Yes & $\mathrm{R} 1 ; Z$ & Seismograms & $3.3,4.1$ \\
9 & generic & Flat & Yes & $\mathrm{R} 1 ; N$ & Seismograms & 4.2 \\
10 & generic & Topo $20 \mathrm{~m}$ & Yes & $\mathrm{R} 1 ; N$ & Seismograms & 4.2 \\
11 & generic & Topo $20 \mathrm{~m}$ & No & $\mathrm{R} 1 ; Z$ & Snapshots & 3.4 \\
\hline 12 & generic & Flat & Yes & $\mathrm{S} 1 ; Z$ & Seismograms & 4.3 \\
13 & generic & Flat rough & Yes & $\mathrm{S} 1 ; Z$ & Seismograms & 4.3 \\
14 & generic & Craters & Yes & $\mathrm{S} 1 ; Z$ & Seismograms & 4.3 \\
\hline 15 & generic & Flat & Yes & reciprocal & Seismograms & $5.2 .1,5.3 .2$ \\
16 & generic & Topo $10 \mathrm{~m}$ & Yes & reciprocal & Seismograms & $3.3,5.2 .2,5.3 .2$ \\
\hline
\end{tabular}

${ }^{c}$ Configurations according to velocity model, surface characteristics, attenuation ' $\alpha$ ', source position and direction, and output, along with indication of the section of the present article in which each configuration is referred to. 'Topo $20 \mathrm{~m}$ ' and 'Topo 10m' refers to topography with $20 \mathrm{~m}$ and $10 \mathrm{~m}$ resolution, respectively. 'R1' corresponds to source position of rockfall 1, Figure 1b; 'S1' to the source position of the synthetic crater study, Figure 7. Reciprocal simulations are carried out for each seismometer (BON, BOR, DSO, SNE) and for each direction $(E, N, Z)$. 

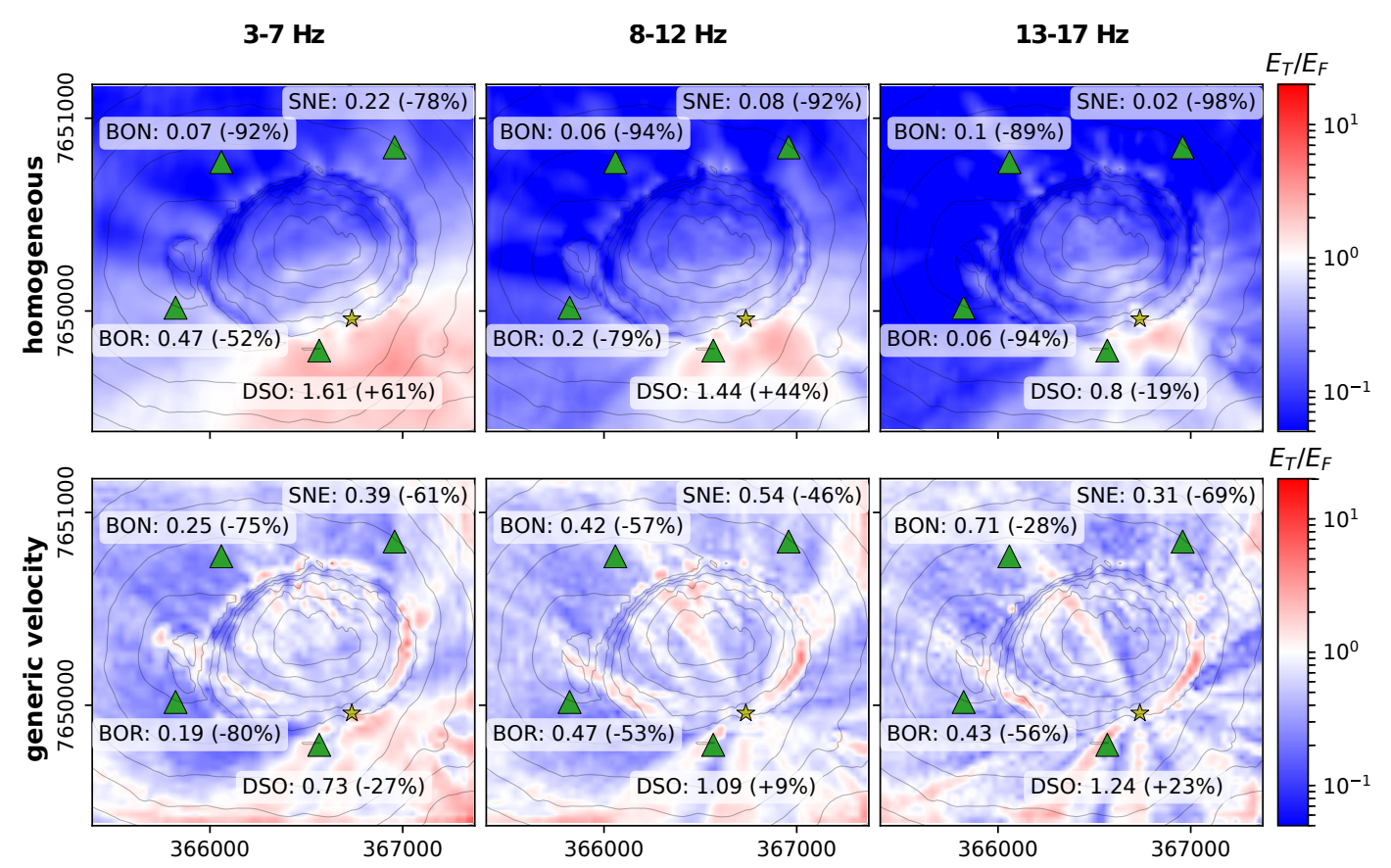

\section{Appendix B Energy amplification in different frequency bands}

Figure B1 shows energy amplification in three different frequency bands for the homogeneous velocity model (top) and the generic velocity model (bottom). Rayleigh wavelengths in frequency band $3-7 \mathrm{~Hz}$ of the homogeneous model $\left(\lambda \approx 1000 \mathrm{~m} \mathrm{~s}^{-1} \div 10 \mathrm{~Hz}=\right.$ $100 \mathrm{~m}$ ) are comparable to those in frequency band $8-12 \mathrm{~Hz}$ of the Lesage generic model $\left(\lambda \approx 580 \mathrm{~m} \mathrm{~s}^{-1} \div 5 \mathrm{~Hz} \approx 116 \mathrm{~m}\right.$, see dispersion curves in Figure $\left.2 \mathrm{c}\right)$. However, we can observe that the amplification patterns differ in these two frequency bands. This suggests that the respective amplification patterns are not only characteristic of a certain wavelength. The wave propagation essentially depends on the velocity model which hence results in different amplification patterns.

Figure B1. Amplification of total kinetic energy in frequency bands 3-7 Hz (left), 8-12 Hz (middle) and 13-17 Hz (right) for the homogeneous velocity model (top) and the Lesage generic velocity model (bottom). The yellow star denotes the source and the green triangles the stations. Annotations indicate ratios measured at the station locations as well as the percentage of topographic amplification. Neighboring contour lines differ by $60 \mathrm{~m}$ in elevation.

\section{Appendix C Estimation of site effects caused by local subsurface struc- tures}

Before calculating the site amplification functions, Figure C1a compares spectral ratios from the uncorrected rockfall signals to those from simulations with the models using the Dolomieu topography. Note that the ratios from the simulations seem to be smaller than the real values. In particular ratio SNE/BON is strongly underestimated, especially for the horizontal components. This is possibly caused by local structures in the subsurface which are not accounted for in the simulations. Local site effects are estimated to correct the recorded signals and ensure comparability between observations and simulations.

Site effects are estimated from seismic signals generated by volcano-tectonic (VT) events which are centered around $2 \mathrm{~km}$ below Dolomieu crater. Thirty-six events are se- 
a)
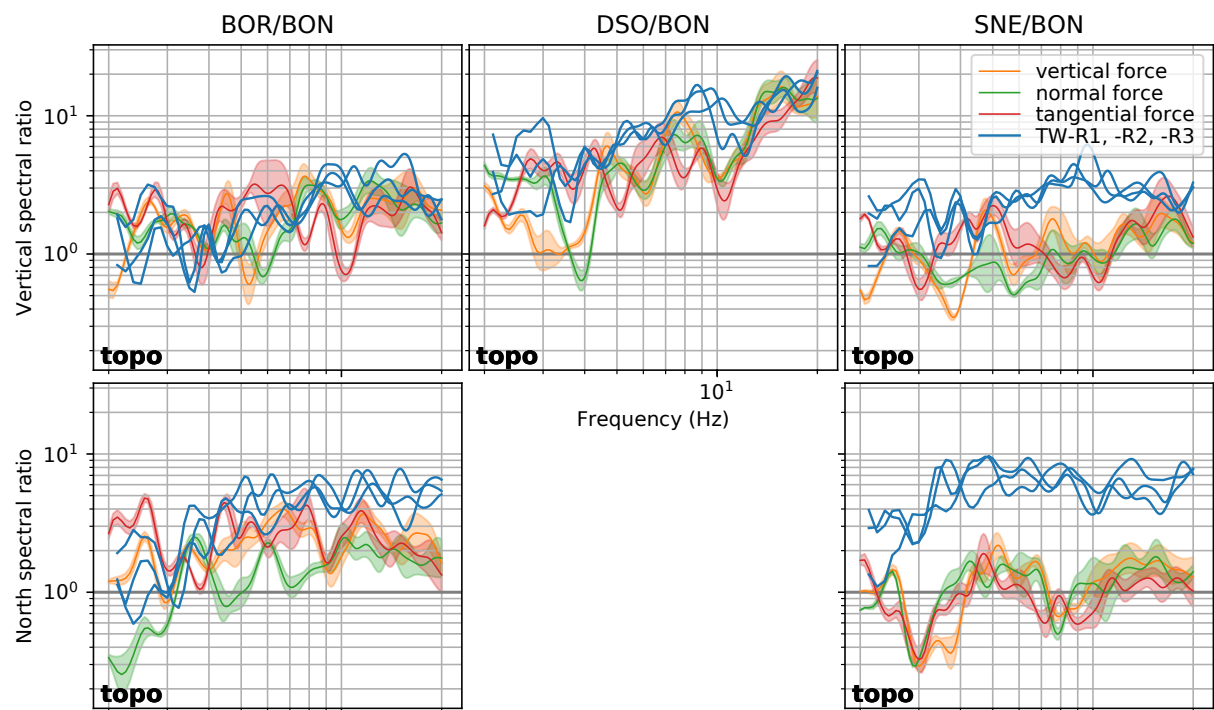

Frequency $(\mathrm{Hz})$
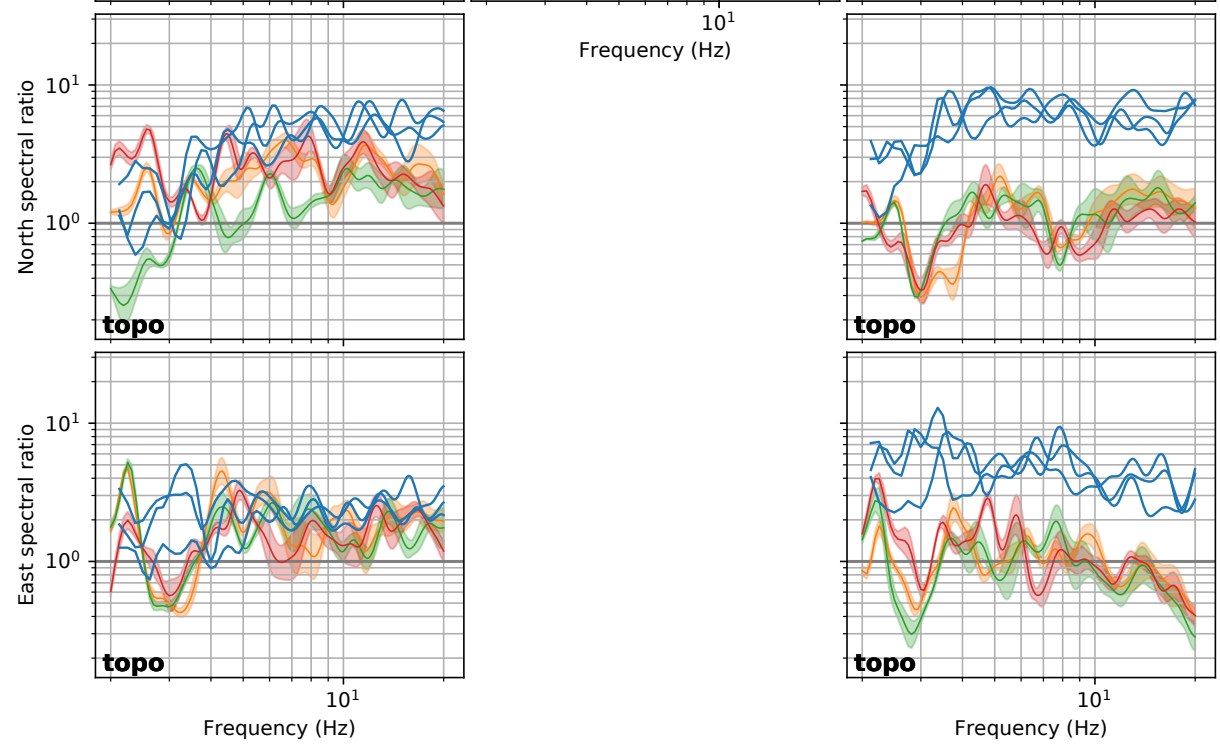

b)

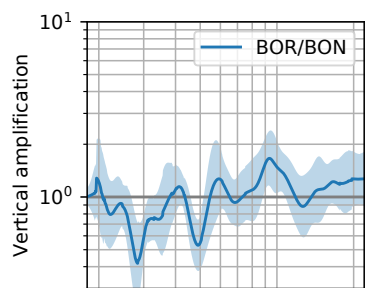

$10^{1}$

Frequency $(\mathrm{Hz})$
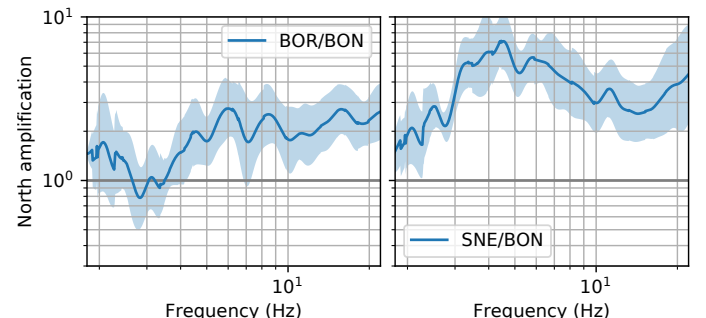

Frequency $(\mathrm{Hz})$

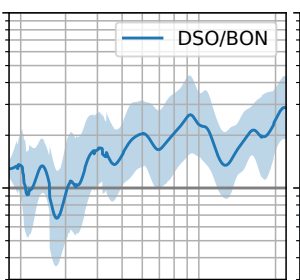

$10^{1}$

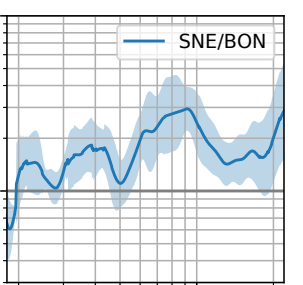

Frequency $\stackrel{10^{1}}{(\mathrm{~Hz})}$
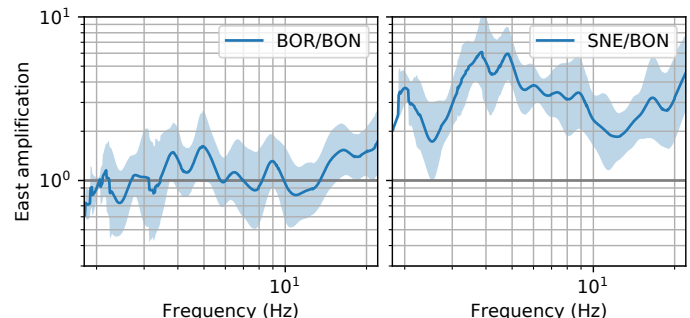

Frequency $(\mathrm{Hz})$

Figure C1. a) Comparison of spectral ratios as in Figure 12 but with before site effect correction of the recorded signals (blue). This leads to partly strong deviations between observations and simulations. b) Spectral amplification functions relative to reference BON for ground velocity of vertical (top), north (bottom left) and east (bottom right) component. The blue-shaded zone indicates the standard deviation of the amplification distribution from all VTs.

lected from a catalog compiled by Duputel et al. (2019). To compute the amplification functions, BON was qualified as an adequate reference station based on the low spec- 
tral amplitudes of both VT signals and $\mathrm{H} / \mathrm{V}$ noise ratios. The spectral ratios are computed from FFT spectra after applying the smoothing function proposed by Konno and Ohmachi (1998) to avoid spurious fluctuations. Figure C1b shows the mean spectral amplification functions and their standard deviation calculated from all VT events for all components.

Strongest amplification is experienced by station SNE with factors up to 7 on its horizontal components. This can explain the strong mismatch between observations and simulations that are visible in Figure C1a. The vertical component of single-component station DSO also seems to be amplified with a peak around $9 \mathrm{~Hz}$. Less evidence of amplification is found for station BOR, except for its north-component which is amplified by a factor of 2 for frequencies above $5 \mathrm{~Hz}$.

\section{Appendix D Observed and simulated spectral ratios for rockfall sources in the southwest}

To reinforce the findings of section 5.2.2 that spectral ratios are characteristic of the source position and can be reproduced when surface topography is taken into account, the same analysis is carried out here for rockfall sources located on the southwestern crater wall. Snapshots taken from camera CBOC of the three observed rockfalls are shown in Figure D1, together with the generated seismic signals recorded on the vertical components. For the times of the shown images, marked on the seismic signals by the vertical dotted red lines R1, R2, and R3, all the rockfalls are located in the same area.

The camera images reveal that each of the rockfalls involves at least two boulders moving downslope simultaneously. While the boulders of rockfall 1 originate from below the camera position, boulders of rockfall $\mathbf{2}$ and $\mathbf{3}$ come from the right-hand border of the image. At the time of the snapshot, the trajectories of the three rockfalls cross. From a window of $\pm 4 \mathrm{~s}$ around this time, the spectral ratios are computed from the observed signals and shown in Figure D2 (blue, site-effect corrected). As for the rockfalls analyzed above, the spectral ratios from the three events in the southwest are very similar to each other across the whole frequency range for all station pairs.

The spectral ratios are now compared to simulations using the model with Dolomieu topography. As above, three input force configurations are tested (i.e. a vertical force, a force normal to the slope and a force tangential to the slope in direction of the strongest gradient) to investigate the dependency of the ratios on the source direction.

We can generally observe that the simulated spectral ratios agree very well with the observed spectral ratios. Changing the source direction does not essentially influence the spectral ratios, except for frequencies below $5 \mathrm{~Hz}$, which is similar to the observations in Figure 12. The similarity at higher frequencies suggests that the ratios are dominated by propagation along the topography rather than by the source mechanism. The strongest deviation between observations and simulations at high frequencies is visible on ratio SNE/BON for the east-component. In comparison with the observed spectral ratios, the simulated amplitudes measured at station SNE are strongly underestimated with respect to station BON. This could be caused either by soil heterogeneities, which are not considered in the simulations, or by uncertainties of the source position and the fact that the rockfall contains at least two boulders which simultaneously impact the ground.

Analysis of the rockfalls located in the southwestern part of Dolomieu crater supports the findings of section 5.2.2 indicating that the spectral ratios are characteristic of the source location, and can be reproduced by taking into account the surface topography, while source direction is not dominant, in particular at high frequencies. 
1)
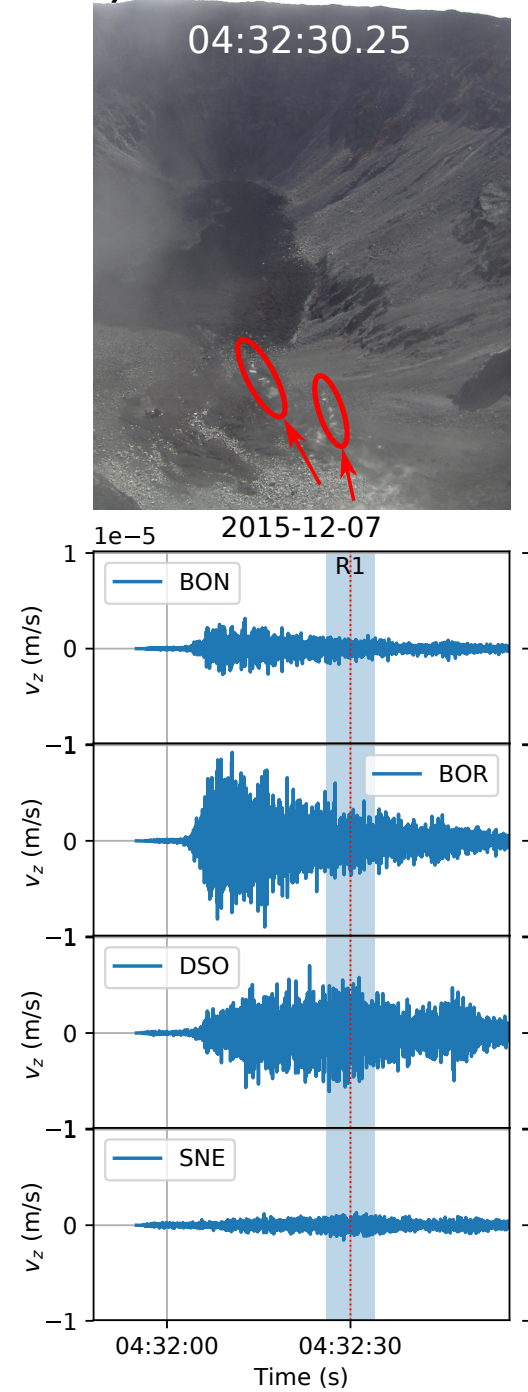

2)

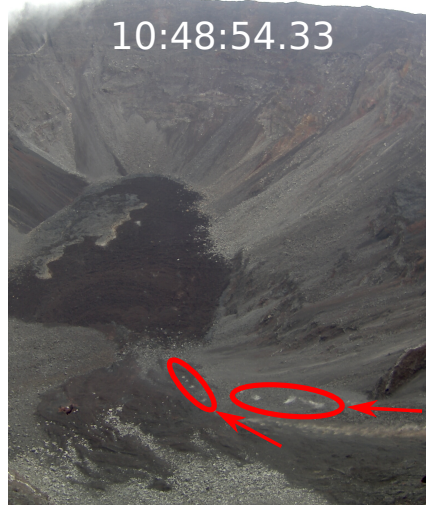

2015-12-07

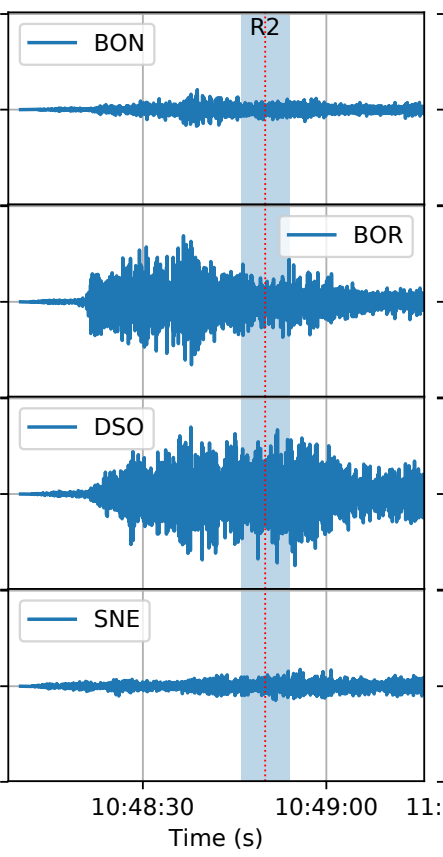

3)

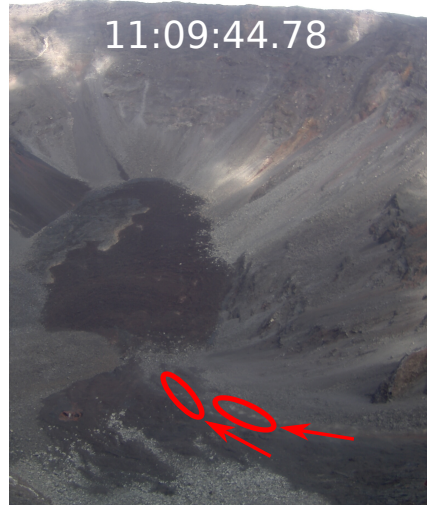

2016-12-13

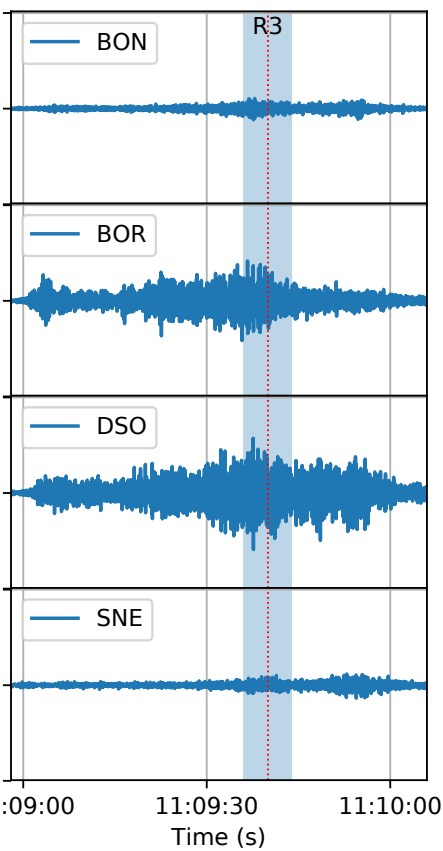

Figure D1. Top: Snapshots taken from camera CBOC of three rockfalls times for which all the rockfalls are in comparable locations. Positions and directions of the boulders are indicated by red circles and arrows. The trajectory of the rockfall on 13 December, 2016 is indicated as event $\mathbf{2}$ on the map in Figure 1b Bottom: Corresponding seismic signals (vertical velocity). The vertical dotted lines R1, R2 and R3 mark the time of the camera snapshot shown above. The blue-shaded zones display the time windows of $\pm 4 \mathrm{~s}$ around R1, R2, and R3 in which spectral station ratios of the signals are computed. 

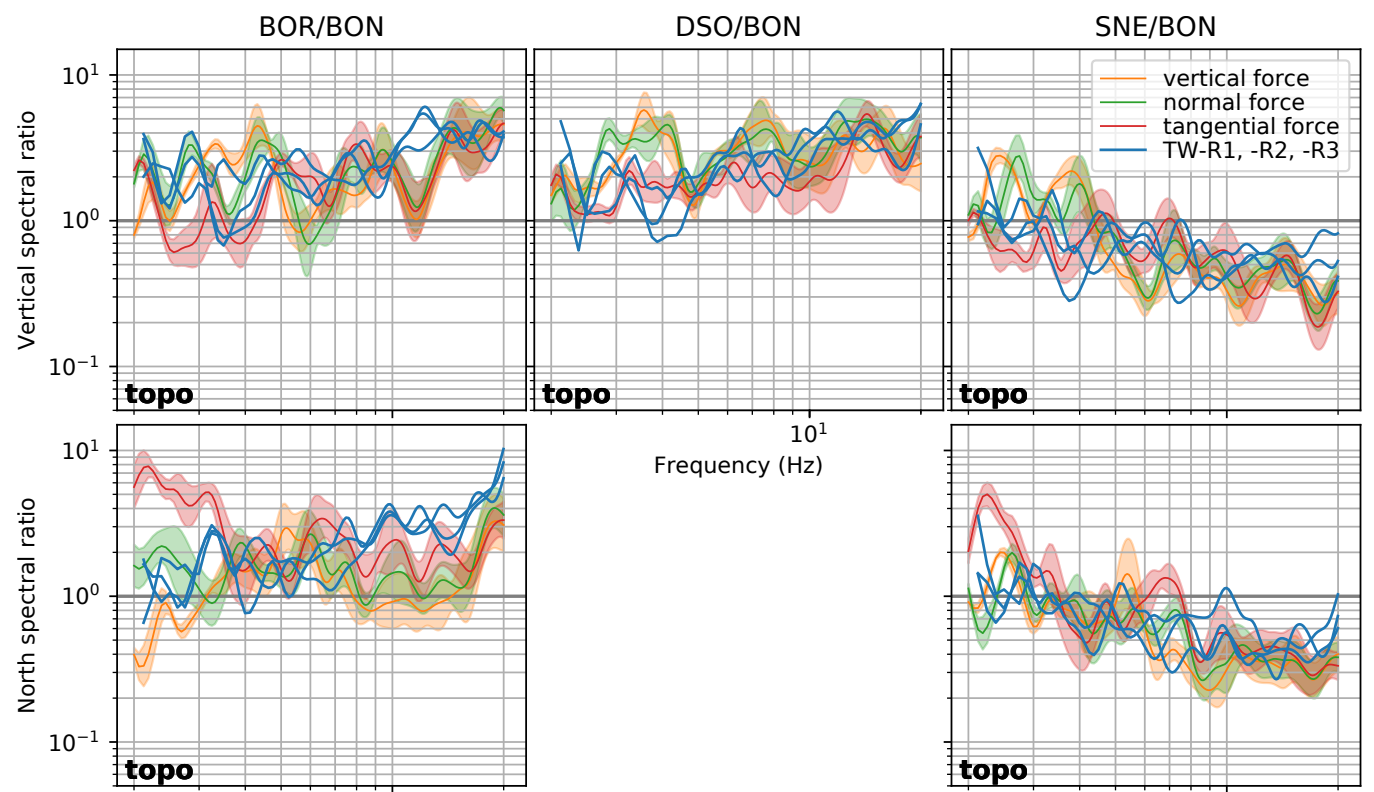

Frequency $(\mathrm{Hz})$
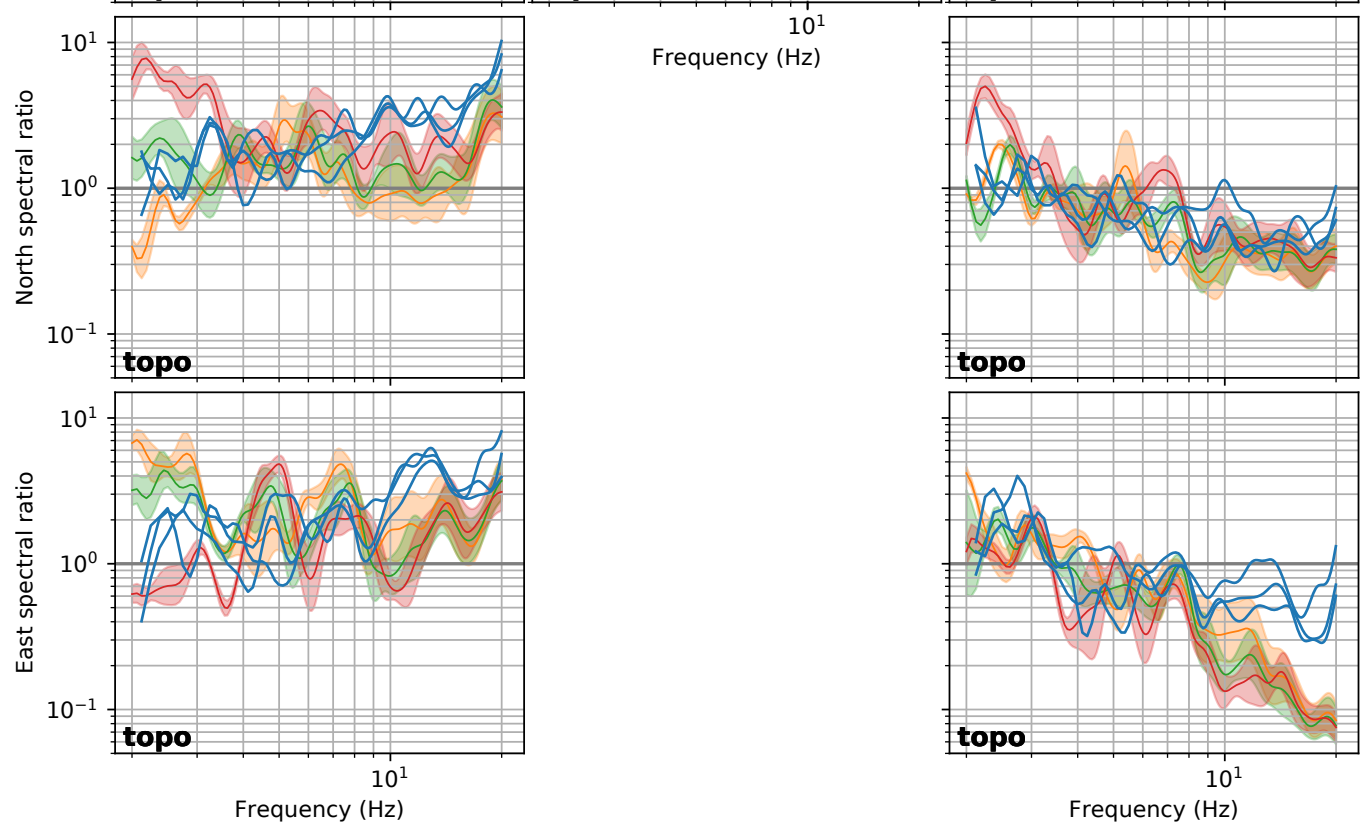

Figure D2. Spectral ratios BOR/BON, DSO/BON and SNE/BON calculated from real signals TW-R1, -R2, -R3 (as defined in Fig. D1) and from simulations on the domain with Dolomieu topography for the vertical- (top), the north- (middle), and the east- (bottom) component. Simulations are carried out with varying source directions: vertical force, force normal to the slope and force tangential to the slope. The shaded zones of the simulated ratios indicate the standard deviation around the mean value of seventeen neighbouring source positions located close to index number $\mathbf{2}$ on the trajectory of event $\mathbf{2}$ on the map in Figure $1 \mathrm{~b}$. 


\section{Acknowledgments}

We would like to thank Jean-Pierre Vilotte and Hugo Martin for fruitful ideas. We are very grateful to Victor Tsai and an anonymous reviewer for their critical and constructive remarks which substantially contributed to improve the quality of the paper. We thank the whole team at the OVPF that provided the excellent field data for this study. The seismic data used in this study, were acquired by the Volcanological and Seismological Observatory of Piton de la Fournaise (OVPF)/Institut de Physique du Globe de Paris (IPGP) via the VOLOBSIS Portal: http://volobsis.ipgp.fr/query.php. Camera data are stored in http://doi.org/10.5281/zenodo.4031816. Data from the simulations are available from http://doi.org/10.5281/zenodo.3949826. Numerical computations were partly performed at S-CAPAD (Service de calcul parallèle et de traitement de données en sciences de la Terre), IPGP, France, as well as at CCIPL (Centre de Calcul Intensif des Pays de la Loire), Université de Nantes, France. This work was funded by the ERC Contract No. ERC-CG-2013-PE10-617472 SLIDEQUAKES.

\section{References}

Ancheta, T. D., Darragh, R. B., Stewart, J. P., Seyhan, E., Silva, W. J., Chiou, B. S., ... Donahue, J. L. (2014). NGA-West2 database. Earthquake Spectra, 30 (3), 989-1005. doi: 10.1193/070913EQS197M

Assimaki, D., \& Jeong, S. (2013). Ground-motion observations at hotel Montana during the M 7.0 2010 Haiti earthquake: Topography or soil amplification? Bulletin of the Seismological Society of America, 103(5), 2577-2590. doi: 10.1785/0120120242

Bachelet, V., Mangeney, A., De Rosny, J., Toussaint, R., \& Farin, M. (2018). Elastic wave generated by granular impact on rough and erodible surfaces. Journal of Applied Physics, 123(4). doi: 10.1063/1.5012979

Battaglia, J. (2003). Location of seismic events and eruptive fissures on the Piton de la Fournaise volcano using seismic amplitudes. Journal of Geophysical Research, 108(B8), 2364. Retrieved from http://doi.wiley.com/10.1029/ 2002JB002193 doi: 10.1029/2002jb002193

Bettuzzi, S. (2009). Introduction (Vol. 104) (No. 1). University Science Books, Sausalito, CA. doi: 10.1016/S0065-230X(09)04001-9

Bottelin, P., Jongmans, D., Daudon, D., Mathy, A., Helmstetter, A., BonillaSierra, V., ... Donzé, F. (2014). Seismic and mechanical studies of the artificially triggered rockfall at Mount Néron (French Alps, December 2011). Natural Hazards and Earth System Sciences, 14(12), 3175-3193. doi: 10.5194/nhess-14-3175-2014

Bouchon, M., \& Barker, J. S. (1996). Seismic response of a hill: The example of Tarzana, California. Bulletin of the Seismological Society of America, 86(1 SUPPL. A), 66-72.

Chaljub, E., Komatitsch, D., Vilotte, J. P., Capdeville, Y., Valette, B., \& Festa, G. (2007). Spectral-element analysis in seismology. Advances in Geophysics, 48(06), 365-419. doi: 10.1016/S0065-2687(06)48007-9

Chiou, B. S. J., \& Youngs, R. R. (2014). Update of the Chiou and Youngs NGA model for the average horizontal component of peak ground motion and response spectra. Earthquake Spectra, 30(3), 1117-1153. doi: 10.1193/072813EQS219M

Dammeier, F., Moore, J. R., Haslinger, F., \& Loew, S. (2011, nov). Characterization of alpine rockslides using statistical analysis of seismic signals. Journal of Geophysical Research: Earth Surface, 116(4), F04024. Retrieved from http://doi .wiley.com/10.1029/2011JF002037 doi: 10.1029/2011JF002037

Davis, L. L., \& West, L. R. (1973). Observed effects of topography on ground motion. Bulletin of the Seismological Society of America, 63(1), 747-763.

Deparis, J., Jongmans, D., Cotton, F., Baillet, L., Thouvenot, F., \& Hantz, D. 
(2008, aug). Analysis of rock-fall and rock-fall avalanche seismograms in the French Alps. Bulletin of the Seismological Society of America, 98(4), 17811796. Retrieved from https://pubs.geoscienceworld.org/bssa/article/ 98/4/1781-1796/341946http://www.bssaonline.org/cgi/doi/10.1785/ 0120070082 doi: 10.1785/0120070082

Derrien, A., Villeneuve, N., Peltier, A., \& Michon, L. (2019). Multi-temporal airborne structure-from-motion on caldera rim: Hazard, visitor exposure and origins of instabilities at Piton de la Fournaise. Progress in Physical Geography, 43(2), 193-214. doi: 10.1177/0309133318808201

Dewez, T. J., Nachbaur, A., Mathon, C., Sedan, O., Kobayashi, H., Rivière, C., ... Nowak, E. (2010). OFAI: 3D block tracking experiment on a weathered volcanic rock slope of Tahiti, French Polynesia. Conf. Procedings, Rock Slope Stability 2010, 24-25 Nov. 2010, Paris, France.

Duputel, Z., Lengliné, O., \& Ferrazzini, V. (2019). C Constraining Spatiotemporal Characteristics of Magma Migration at Piton De La Fournaise Volcano From Pre-eruptive Seismicity. Geophysical Research Letters, 46(1), 119-127. doi: 10.1029/2018GL080895

Durand, V., Mangeney, A., Haas, F., Jia, X., Bonilla, F., Peltier, A., ... Villeneuve, N. (2018, oct). On the Link Between External Forcings and Slope Instabilities in the Piton de la Fournaise Summit Crater, Reunion Island. Journal of Geophysical Research: Earth Surface, 123(10), 24222442. Retrieved from http://doi.wiley.com/10.1029/2017JF004507 doi: 10.1029/2017JF004507

Farin, M., Mangeney, A., Toussaint, R., Rosny, J. D., Shapiro, N., Dewez, T., ... Berger, F. (2015, oct). Characterization of rockfalls from seismic signal: Insights from laboratory experiments. Journal of Geophysical Research: Solid Earth, 120(10), 7102-7137. Retrieved from http://doi.wiley.com/10.1002/ 2015JB012331 doi: 10.1002/2015JB012331

Favreau, P., Mangeney, A., Lucas, A., Crosta, G., \& Bouchut, F. （2010, aug). Numerical modeling of landquakes. Geophysical Research Letters, 37(15), 1-5. Retrieved from http://doi.wiley.com/10.1029/2010GL043512 doi: 10.1029/2010GL043512

Festa, G., \& Vilotte, J. P. (2005, jun). The Newmark scheme as velocity-stress time-staggering: An efficient PML implementation for spectral element simulations of elastodynamics. Geophysical Journal International, 161(3), 789-812. Retrieved from https://academic.oup.com/gji/article-lookup/doi/10 .1111/j.1365-246X.2005.02601.x doi: 10.1111/j.1365-246X.2005.02601.x

Geli, L., Bard, P. Y., \& Jullien, B. (1988). The effect of topography on earthquake ground motion: a review and new results. Bulletin - Seismological Society of America, 78(1), 42-63. doi: 10.1016/0148-9062(88)90024-1

Hailemikael, S., Lenti, L., Martino, S., Paciello, A., Rossi, D., \& Scarascia Mugnozza, G. (2016, jul). Ground-motion amplification at the Colle di Roio ridge, central Italy: A combined effect of stratigraphy and topography. Geophysical Journal International, 206(1), 1-18. Retrieved from https://academic. oup.com/gji/article-lookup/doi/10.1093/gji/ggw120 doi: $10.1093 / \mathrm{gji} / \mathrm{ggw} 120$

Harp, E. L., Hartzell, S. H., Jibson, R. W., Ramirez-Guzman, L., \& Schmitt, R. G. (2014). Relation of landslides triggered by the Kiholo Bay Earthquake to modeled ground motion. Bulletin of the Seismological Society of America, 104(5), 2529-2540. doi: 10.1785/0120140047

Hartzell, S. H., Carver, D. L., \& King, K. W. (1994). Initial investigation of site and topographic effects at Robinwood Ridge, California. Bulletin - Seismological Society of America, 84(5), 1336-1349.

Herrmann, R. B. (2013, nov). Computer programs in seismology: An evolving tool for instruction and research. Seismological Research Letters, 84 (6), 1081-1088. 
Retrieved from https://pubs.geoscienceworld.org/srl/article/84/6/ 1081-1088/315307 doi: 10.1785/0220110096

Hertz, V. H. H. (1878). Die lieber die Berührung fester elastischer Körper. Journal für die reine und angewandte Mathematik, 92, 156-171.

Hibert, C., Ekström, G., \& Stark, C. P. $\quad$ (2014, jul). Dynamics of the Bingham Canyon Mine landslides from seismic signal analysis. Geophysical Research Letters, 41(13), 4535-4541. Retrieved from http://doi.wiley.com/10.1002/ 2014GL060592 doi: 10.1002/2014GL060592

Hibert, C., Malet, J. P., Bourrier, F., Provost, F., Franck Bourrier, Bornemann, P., .. Mermin, E. (2017, may). Single-block rockfall dynamics inferred from seismic signal analysis. Earth Surface Dynamics, 5(2), 283-292. Retrieved from https://www.earth-surf-dynam.net/5/283/2017/ doi: 10.5194/esurf-5-283-2017

Hibert, C., Mangeney, A., Grandjean, G., Baillard, C., Rivet, D., Shapiro, N. M., ... Crawford, W. (2014, may). Automated identification, location, and volume estimation of rockfalls at Piton de la Fournaise volcano. Journal of Geophysical Research: Earth Surface, 119(5), 1082-1105. Retrieved from http:// doi.wiley.com/10.1002/2013JF002970 doi: 10.1002/2013JF002970

Hibert, C., Mangeney, A., Grandjean, G., Peltier, A., DiMuro, A., Shapiro, N. M., ... Kowalski, P. (2017, mar). Spatio-temporal evolution of rockfall activity from 2007 to 2011 at the Piton de la Fournaise volcano inferred from seismic data. Journal of Volcanology and Geothermal Research, 333-334, 36-52. Retrieved from https://www-sciencedirect-com.insu.bib.cnrs.fr/science/ article/pii/S0377027316303195 doi: 10.1016/j.jvolgeores.2017.01.007

Hibert, C., Mangeney, A., Grandjean, G., \& Shapiro, N. M. (2011, dec). Slope instabilities in Dolomieu crater, Réunion Island: From seismic signals to rockfall characteristics. Journal of Geophysical Research: Earth Surface, 116(4), 1-18. Retrieved from http://doi.wiley.com/10.1029/2011JF002038 doi: 10.1029/2011JF002038

Hough, S. E., Altidor, J. R., Anglade, D., Given, D., Janvier, M. G., Maharrey, J. Z., ... Yong, A. (2010). Localized damage caused by topographic amplification during the 2010 M7.0 Haiti earthquake. Nature Geoscience, 3(11), 778-782. Retrieved from http://dx.doi.org/10.1038/ngeo988 doi: $10.1038 /$ ngeo988

Imperatori, W., \& Mai, P. M. (2013). Broad-band near-field ground motion simulations in 3-dimensional scattering media. Geophysical Journal International, 192(2), 725-744. doi: 10.1093/gji/ggs041

Jeong, S., Asimaki, D., Dafni, J., \& Wartman, J. (2019, jan). How topographydependent are topographic effects? Complementary numerical modeling of centrifuge experiments. Soil Dynamics and Earthquake Engineering, 116, 654667. Retrieved from https://www.sciencedirect.com/science/article/ pii/S0267726117310849 doi: 10.1016/j.soildyn.2018.10.028

Johnson, K. L. (1989). Contact Mechanics. Cambridge University Press. Retrieved from https://books.google.fr/books?id=Do6WQlUwbpkC\&pg= PA45\&lpg=PA45\&dq=johnson+1885+contact+mechanics\&source=bl\&ots= gqjfkuec1Y\&sig=ACfU3U3_MGjPLQugBHMiitM1K3uIUZorZw\&hl=en\&sa=X\&ved= 2ahUKEwi3zoSO_I_lAhUF-YUKHfdoBdAQ6AEwBXoECAkQAg\#v=onepage\&q= johnson1885contac doi: 10.1201/b17110-2

Köhler, A., Ohrnberger, M., Scherbaum, F., Wathelet, M., \& Cornou, C. (2007, feb). Assessing the reliability of the modified three-component spatial autocorrelation technique. Geophysical Journal International, 168(2), 779-796. Retrieved from https://academic.oup.com/gji/article-lookup/doi/10.1111/ j.1365-246X.2006.03253.x doi: 10.1111/j.1365-246X.2006.03253.x

Konno, K., \& Ohmachi, T. (1998). Ground-motion characteristics estimated from spectral ratio between horizontal and vertical components of microtremor. Bul- 
letin of the Seismological Society of America, 88(1), 228-241.

Kuehnert, J., Mangeney, A., Capdeville, Y., Vilotte, J. P., \& Stutzmann, E. (2020). Rockfall localization based on inter-station ratios of seismic energy. Earth and Space Science Open Archive, 1-34. doi: 10.1002/ESSOAR.10503303.1

Larose, E., Carrière, S., Voisin, C., Bottelin, P., Baillet, L., Guéguen, P., ... Massey, C. (2015). Environmental seismology: What can we learn on earth surface processes with ambient noise? Journal of Applied Geophysics, 116, 62-74. Retrieved from http://dx.doi.org/10.1016/j.jappgeo.2015.02.001 doi: 10.1016/j.jappgeo.2015.02.001

Lee, S. J., Chan, Y. C., Komatitsch, D., Huang, B. S., \& Tromp, J. (2009). Effects of realistic surface topography on seismic ground motion in the Yangminshan region of Taiwan based upon the spectral-element method and LiDAR DTM. Bulletin of the Seismological Society of America, 99(2 A), 681-693. doi: 10.1785/0120080264

Lee, S. J., Komatitsch, D., Huang, B. S., \& Tromp, J. $\quad$ (2009). $\quad$ Effects of topography on seismic-wave propagation: An example from Northern Taiwan. Bulletin of the Seismological Society of America, 99(1), 314-325. doi: 10.1785/0120080020

Lee, W. H. K., White, R. A., Harlow, D. H., Rogers, J. A., Spudich, P., \& Dodge, D. A. (1994). Digital seismograms of selected aftershocks of the Northridge earthquake recorded by a dense seismic array on February 11, 1994 at Cedar Hill Nursery in Tarzana, California. US Geol. Surv. Open-File Rept. 94, 234.

Lesage, P., Heap, M. J., \& Kushnir, A. (2018). A generic model for the shallow velocity structure of volcanoes. Journal of Volcanology and Geothermal Research, 356,114-126. Retrieved from https://doi.org/10.1016/j.jvolgeores. 2018 .03.003 doi: 10.1016/j.jvolgeores.2018.03.003

Ma, S., Archuleta, R. J., \& Page, M. T. (2007). Effects of large-scale surface topography on ground motions, as demonstrated by a study of the San Gabriel Mountains, Los Angeles, California. Bulletin of the Seismological Society of America, 97(6), 2066-2079. doi: 10.1785/0120070040

Maufroy, E., Cruz-Atienza, V. M., Cotton, F., \& Gaffet, S. (2015). Frequency-scaled curvature as a proxy for topographic site-effect amplification and groundmotion variability. Bulletin of the Seismological Society of America, 105(1), 354-367. doi: 10.1785/0120140089

McLaskey, G. C., \& Glaser, S. D. (2010). Hertzian impact: Experimental study of the force pulse and resulting stress waves. The Journal of the Acoustical Society of America, 128(3), 1087. doi: 10.1121/1.3466847

Meunier, P., Hovius, N., \& Haines, J. A. (2008). Topographic site effects and the location of earthquake induced landslides. Earth and Planetary Science Letters, 275(3-4), 221-232. Retrieved from http://dx.doi.org/10.1016/j.epsl.2008 .07 .020 doi: $10.1016 /$ j.epsl.2008.07.020

Mordret, A., Rivet, D., Landès, M., \& Shapiro, N. M. (2015). Three-dimensional shear velocity anisotropic model of Piton de la Fournaise Volcano (La Réunion Island) from ambient seismic noise. Journal of Geophysical Research: Solid Earth, 120(1), 406-427. doi: 10.1002/2014JB011654

Moretti, L., Mangeney, A., Capdeville, Y., Stutzmann, E., Huggel, C., Schneider, D., \& Bouchut, F. (2012, aug). Numerical modeling of the Mount Steller landslide flow history and of the generated long period seismic waves. Geophysical Research Letters, 39(16), 1-7. Retrieved from http://doi.wiley.com/10.1029/ 2012GL052511 doi: 10.1029/2012GL052511

Munasinghe, M., \& Farnell, G. W. (1973). Finite difference analysis of rayleigh wave scattering at vertical discontinuities. Journal of Geophysical Research, 78(14), 2454-2466. doi: 10.1029/jb078i014p02454

O'Brien, G. S., \& Bean, C. J. (2009). Volcano topography, structure and intrinsic attenuation: Their relative influences on a simulated $3 \mathrm{D}$ visco-elastic wave- 
field. Journal of Volcanology and Geothermal Research, 183(1-2), 122-136. Retrieved from http://dx.doi.org/10.1016/j.jvolgeores. 2009.03.004 doi: 10.1016/j.jvolgeores.2009.03.004

Pedersen, H., Le Brun, B., Hatzfeld, D., Campillo, M., \& Bard, P. Y. Ground-motion amplitude across ridges. Bulletin - Seismological Society of America, 84 (6), 1786-1800. Retrieved from http://www.bssaonline.org/ content/84/6/1786. short\%5Cn\%3CGotoISI\%3E: //WOS: A1994QA09700006

Rai, M., Rodriguez-Marek, A., \& Chiou, B. S. (2017). Empirical Terrain-Based Topographic Modification Factors for Use in Ground Motion Prediction. Earthquake Spectra, 33(1), 157-177. doi: 10.1193/071015EQS111M

Sánchez-Sesma, F. J., \& Campillo, M. (1993). Topographic effects for incident P, SV and Rayleigh waves. Tectonophysics, 218(1-3), 113-125. doi: 10.1016/0040 -1951(93)90263-J

Snieder, R. (1986). The influence of topography on the propagation and scattering of surface waves. Physics of the Earth and Planetary Interiors, 44 (3), 226-241. doi: 10.1016/0031-9201(86)90072-5

Soontiens, N., Stastna, M., \& Waite, M. L. $\quad$ (2013, apr). Numerical simulations of waves over large crater topography in the atmosphere. Journal of the Atmospheric Sciences, 70(4), 1216-1232. Retrieved from http://journals.ametsoc.org/doi/abs/10.1175/JAS-D-12-0221.1 doi: 10.1175/JAS-D-12-0221.1

Spudich, P., Hellweg, M., \& Lee, W. H. (1996). Directional topographic site response at Tarzana observed in aftershocks of the 1994 Northridge, California, earthquake: Implications for mainshock motions. Bulletin of the Seismological Society of America, 86(1 SUPPL. B), 193-208.

Staudacher, T., Ferrazzini, V., Peltier, A., Kowalski, P., Boissier, P., Catherine, P., ... Massin, F. (2009). The April 2007 eruption and the Dolomieu crater collapse, two major events at Piton de la Fournaise (La Réunion Island, Indian Ocean). Journal of Volcanology and Geothermal Research, 184(1-2), 126-137. Retrieved from http://dx.doi.org/10.1016/j.jvolgeores.2008.11.005 doi: $10.1016 /$ j.jvolgeores.2008.11.005

Vilajosana, I., Suriñach, E., Abellán, A., Khazaradze, G., Garcia, D., \& Llosa, J. (2008, aug). Rockfall induced seismic signals: Case study in Montserrat, Catalonia. Natural Hazards and Earth System Science, 8(4), 805-812. Retrieved from https://www.nat-hazards-earth-syst-sci.net/8/805/2008/ doi: 10.5194/nhess-8-805-2008

Volkwein, A., Schellenberg, K., Labiouse, V., Agliardi, F., Berger, F., Bourrier, F., ... Jaboyedoff, M. (2011). Rockfall characterisation and structural protection - A review. $\quad$ Natural Hazards and Earth System Science, 11 (9), 2617-2651. Retrieved from https://hal.archives-ouvertes.fr/hal-00653458/ doi: 10.5194/nhess-11-2617-2011

Wang, B., Da, Y., \& Qian, Z. (2018). Forward and inverse studies on scattering of Rayleigh wave at surface flaws. Applied Sciences (Switzerland), 8(3), 427. Retrieved from http://www.mdpi.com/2076-3417/8/3/427 doi: 10.3390/app8030427

Wang, L., Xu, Y., Xia, J., \& Luo, Y. (2015). Effect of near-surface topography on high-frequency Rayleigh-wave propagation. Journal of Applied Geophysics, 116, 93-103. Retrieved from http://dx.doi.org/10.1016/j.jappgeo.2015 .02.028 doi: $10.1016 /$ j.jappgeo.2015.02.028

Wathelet, M., Jongmans, D., Ohrnberger, M., \& Bonnefoy-Claudet, S. $\quad$ (2008, jan). Array performances for ambient vibrations on a shallow structure and consequences over Vs inversion. Journal of Seismology, 12(1), 1-19. Retrieved from http://link.springer.com/10.1007/s10950-007-9067-x doi: 10.1007/s10950-007-9067-x

Weaver, R. L. (1982). on Diffuse Waves in Solid Media. Proceedings of the U.S. Na- 
1279

1280

1281

1282

1283

1284

tional Congress of Applied Mechanics, $71(6), 471$.

Zhang, Z., Fleurisson, J. A., \& Pellet, F. (2018). The effects of slope topography on acceleration amplification and interaction between slope topography and seismic input motion. Soil Dynamics and Earthquake Engineering, 113(May), 420-431. Retrieved from https://doi.org/10.1016/j.soildyn.2018.06.019 doi: $10.1016 /$ j.soildyn.2018.06.019 